CIRJE-F-902

\title{
On an Asymptotic Expansion of Forward-Backward SDEs with a Perturbed Driver
}

\author{
Akihiko Takahashi \\ The University of Tokyo \\ Toshihiro Yamada \\ Graduate School of Economics, The University of Tokyo \& \\ Mitsubishi UFJ Trust Investment Technology Institute Co. Ltd. (MTEC)
}

September 2013; Revised in October 2013

CIRJE Discussion Papers can be downloaded without charge from:

http://www.cirje.e.u-tokyo.ac.jp/research/03research02dp.html

Discussion Papers are a series of manuscripts in their draft form. They are not intended for circulation or distribution except as indicated by the author. For that reason Discussion Papers may not be reproduced or distributed without the written consent of the author. 


\title{
On an Asymptotic Expansion of Forward-Backward SDEs with a Perturbed Driver
}

\author{
Akihiko Takahashi* and Toshihiro Yamada ${ }^{\dagger}$
}

October 16, 2013

\begin{abstract}
This paper presents a mathematical validity for an asymptotic expansion scheme of the solutions to the forwardbackward stochastic differential equations (FBSDEs) in terms of a perturbed driver in the BSDE and a small diffusion in the FSDE. This computational scheme was proposed by Fujii and Takahashi (2012a), which has been successfully employed to solve the derivatives and optimal portfolio problems in Fujii and Takahashi (2012b,c) and Fujii et al. (2012). In particular, we represent the coefficients up to an arbitrary order expansion of the BSDE by the solution to a system of the associated BSDEs with the FSDE, and obtain the error estimate of the expansion with respect to the driver perturbation. Accordingly, we show a concrete representation for each expansion coefficient of the volatility component, that is the martingale integrand in the BSDE. Then, we apply our proposed FSDE expansion formula with its precise error estimate to the BSDE expansion coefficients to finally obtain the total residual estimate.
\end{abstract}

Keywords: Forward-Backward SDEs, Asymptotic expansion, Malliavin calculus, Kusuoka-Stroock functions

\section{Introduction}

This paper investigates the mathematical foundation for an asymptotic expansion scheme of the forward-backward SDEs (FBSDEs) with a perturbed driver proposed by Fujii and Takahashi (2012a). In particular, we concentrate on to provide a mathematical validity for the decoupled case of the scheme, which is explained to the detail in their paper.

The FBSDEs has become quite popular in finance community since El Karoui et al. (1997), especially after the recent financial crises and the subsequent quite volatile markets, which leads us to recognize the importance of counter party risk management, particularly the credit value adjustments (CVA).

However, an explicit solution for a FBSDE has been known only for a simple linear or quadratic example. Although several techniques have been proposed in the last decade, they seem very limited in practical applications since they rely on numerical methods for non-linear PDEs or regression based Monte Carlo simulations, which are generally very difficult to implement or quite time-consuming especially for high-dimensional and long-horizon problems.

Recently, Fujii and Takahashi (2012a) has developed a simple analytical approximation scheme for the nonlinear FBSDEs. They have introduced a perturbation parameter to the driver of a BSDE to expand recursively the nonlinear terms around a relevant linear FBSDE. In the computation of each order, we explicitly represent the backward elements as the functions of the forward components and take those expectations. Hence, except the cases that the distributions of the forward process are explicitly known, we apply some approximations of the distributions such as an asymptotic expansion technique, which is widely applied to the analytical approximations for pricing European contingent claims and computing optimal portfolios.(For example, see Fujii and Takahashi (2012a,b), Takahashi and Yamada $(2012,2013)$ and references therein for the details.)

They also provided two numerical examples, where the second-order analytic approximations work quite well compared to numerical techniques such as the finite difference method and the regression-based Monte Carlo simulation.

Moreover, their subsequent work (Fujii and Takahashi (2012b)) has applied this scheme to the optimal portfolio problem in an incomplete market with stochastic volatility, and demonstrated the accurate approximations even for long maturities such as 10 years, as opposed to the regression based Monte Carlo simulation that works well only up to short maturities such as one year. We also note that the method has the great advantage of deriving explicit expressions of the optimal portfolios and hedging strategies, that is very important in practice. Further, we can use the method for the general multi-dimensional cases, which is not true of the well-know Cole-Hopf transformation. As for the recent development of this scheme with interacting particle method, see Fujii and Takahashi (2012c) and Fujii et al. (2013).

*University of Tokyo

${ }^{\dagger}$ University of Tokyo \& Mitsubishi UFJ Trust Investment Technology Institute Co.. Ltd. (MTEC) 
In a different stream, Takahashi and Yamada (2012) has proposed a new closed-form approximation for the solutions of FBSDEs. In particular, applying Malliavin calculus approach of Kusuoka (2003) and Takahashi and Yamada $(2012,2013)$ to the forward SDEs with the Picard-iteration scheme for the BSDEs, they have obtained an error estimate for the approximation. Moreover, they have demonstrated the effectiveness of the method through numerical examples for pricing options with counter party risk under the local and stochastic volatility models, where the credit value adjustment (CVA) is taken into account.

This paper provides a mathematical foundation for the original scheme in the decoupled case proposed in Fujii and Takahashi (2012a). (The justification for the coupled case will be one of our next research topics.) It mainly consists of two parts. That is, for the BSDE expansion with a perturbed driver we obtain the coefficients up to an arbitrary order as the solution to a system of the associated BSDEs with the base FSDE, and present the error estimate of the expansion. Accordingly, we show a concrete representation for each expansion coefficient of the volatility component, that is the martingale integrand in the BSDE. For the FSDE expansion, we derive an expansion formula with its sharp error estimate for the expectation of the solution to the base FSDE in terms of a small diffusion. Then, we combine the both results, particularly applying our FSDE expansion formula to the BSDE expansion coefficients to obtain our main result, that is an asymptotic expansion of FBSDEs with a perturbed driver. In the proofs, we effectively apply the representation results in Ma and Zhang (2002) for the BSDE expansion and the properties of the Kusuoka-Stroock functions in Kusuoka (2003) for the FSDE expansion.

The organization of the paper is as follows: after the next section describes the basic setup, Section 3 provides the result for the expansion of the BSDE with respect to a perturbation parameter in the driver. Section 4 shows an expansion for the FSDE in terms of a small diffusion, which is combined with the asymptotic expansion for the BSDE in Section 3 to present our main result in Section 5.

\section{FBSDE}

Let $(\Omega, \mathcal{F}, P)$ be a complete probability space on which a $d$-dimensional Brownian motion $W$ is defined. Let $\mathbf{F}=\left\{\mathcal{F}_{t}\right\}$ be the natural filtration generated by $W$, augmented by the $P$-null sets of $\mathcal{F}$. We first consider the following $d$-dimensional forward stochastic differential equation with parameter $\varepsilon,\left(X_{t}^{\varepsilon}\right)_{t}$ with $X_{t}^{\varepsilon}=\left(X_{t}^{\varepsilon, 1}, \cdots, X_{t}^{\varepsilon, d}\right)$ :

$$
d X_{t}^{\varepsilon, i}=b^{i}\left(t, X_{t}^{\varepsilon}\right) d t+\varepsilon \sum_{j=1}^{d} \sigma_{j}^{i}\left(t, X_{t}^{\varepsilon}\right) d W_{t}^{j}, \quad i=1, \cdots, d,
$$

where $b:[0, T] \times \mathbf{R}^{d} \rightarrow \mathbf{R}^{d}, \sigma:[0, T] \times \mathbf{R}^{d} \rightarrow \mathbf{R}^{d \times d}$ and $\varepsilon \in(0,1]$.

Next, we introduce the associated BSDE with a perturbation parameter $\alpha \in[0,1]$ as follows:

$$
Y_{t}^{\alpha, \varepsilon}=g\left(X_{T}^{\varepsilon}\right)+\alpha \int_{t}^{T} f\left(s, X_{s}^{\varepsilon}, Y_{s}^{\alpha, \varepsilon}, Z_{s}^{\alpha, \varepsilon}\right) d s-\int_{t}^{T} Z_{s}^{\alpha, \varepsilon} \cdot d W_{s},
$$

or equivalently, as the differential form:

$$
\begin{aligned}
d Y_{t}^{\alpha, \varepsilon} & =-\alpha f\left(t, X_{t}^{\varepsilon}, Y_{t}^{\alpha, \varepsilon}, Z_{t}^{\alpha, \varepsilon}\right) d t+Z_{t}^{\alpha, \varepsilon} \cdot d W_{t}, \\
Y_{T}^{\alpha, \varepsilon} & =g\left(X_{T}^{\varepsilon}\right),
\end{aligned}
$$

where denote the inner product of $x, y \in \mathbf{R}^{d}$, that is $x \cdot y=\sum_{i=1}^{d} x^{i} y^{i}$ for $\left(x^{1}, \cdots, x^{d}\right)$ and $y=\left(y^{1}, \cdots, y^{d}\right)$.

Then, it holds that

$$
Y_{t}^{\alpha, \varepsilon}=E\left[g\left(X_{T}^{\varepsilon}\right) \mid \mathcal{F}_{t}\right]+\alpha E\left[\int_{t}^{T} f\left(s, X_{s}^{\varepsilon}, Y_{s}^{\alpha, \varepsilon}, Z_{s}^{\alpha, \varepsilon}\right) d s \mid \mathcal{F}_{t}\right] .
$$

We also note that when $\alpha=0, Y_{t}^{0, \varepsilon}$ is the solution to the linear BSDE with $\alpha=0$ in (2):

$$
Y_{t}^{0, \varepsilon}=E\left[g\left(X_{T}^{\varepsilon}\right) \mid \mathcal{F}_{t}\right]
$$

In the following we state the assumptions for the forward-backward SDE in this paper.

\section{Assumption 2.1}

1. The coefficients of the forward process, $b, \sigma$ are bounded Borel functions. Moreover, $b(t, x)$ and $\sigma(t, x)$ are continuous in $(t, x)$ and smooth in $x$ with bounded derivatives of all orders.

2. There exist constants $a_{i}>0, i=1,2$ such that for any vector $\xi$ in $\mathbf{R}^{d}$ and any $(t, x) \in[0, T] \times \mathbf{R}^{d}$,

$$
a_{1}|\xi|^{2} \leq \sum_{i, j=1}^{d}\left[\sigma \sigma^{T}\right]_{i, j}(t, x) \xi_{i} \xi_{j} \leq a_{2}|\xi|^{2}
$$

3. The driver $f:[0, T] \times \mathbf{R}^{d} \times \mathbf{R} \times \mathbf{R}^{d} \rightarrow \mathbf{R}$ is continuous and bounded. Moreover, $f(t, x, y, z)$ is smooth in $x, y, z$ with bounded derivatives of all orders. 
4. $g: \mathbf{R}^{d} \rightarrow \mathbf{R}$ is smooth with bounded derivatives of all orders, and $|g(0)| \leq K$ for a positive constant $K$.

We consider the FBSDEs (1) and (3) on the subinterval $[t, T] \subseteq[0, T]$ as follows: for $s \in[t, T]$,

$$
\begin{aligned}
X_{s}^{t, x, \varepsilon, i} & =x^{i}+\int_{t}^{s} b^{i}\left(r, X_{r}^{t, x, \varepsilon}\right) d r+\varepsilon \sum_{j=1}^{d} \int_{t}^{s} \sigma_{j}^{i}\left(r, X_{r}^{t, x, \varepsilon}\right) d W_{r}^{j} \\
Y_{s}^{t, x, \alpha, \varepsilon} & =g\left(X_{T}^{t, x, \varepsilon}\right)+\alpha \int_{s}^{T} f\left(r, X_{r}^{t, x, \varepsilon}, Y_{r}^{t, x, \alpha, \varepsilon}, Z_{r}^{t, x, \alpha, \varepsilon}\right) d r-\int_{s}^{T} Z_{r}^{t, x, \alpha, \varepsilon} \cdot d W_{r},
\end{aligned}
$$

where the subscript $\cdot^{t, x}$ shows the dependence on the initial data $(t, x)$.

Then, we recall the following well-known result (for instance, see Corollary 4.1 in El Karoui et al. (1997) or Theorem 3.1 in Ma and Zhang (2002).): Define $u^{\alpha, \varepsilon}(t, x)$ as

$$
u^{\alpha, \varepsilon}(t, x):=Y_{t}^{t, x, \alpha, \varepsilon}=E\left[g\left(X_{T}^{t, x, \varepsilon}\right)+\alpha \int_{t}^{T} f\left(r, X_{r}^{t, x, \varepsilon}, Y_{r}^{t, x, \alpha, \varepsilon}, Z_{r}^{t, x, \alpha, \varepsilon}\right) d r\right] .
$$

Then, we have

$$
\partial_{x} u^{\alpha, \varepsilon}(t, x) \sigma(t, x)=Z_{t}^{t, x, \alpha, \varepsilon} .
$$

We also define $\partial_{x} u^{\alpha, \varepsilon} \sigma:[0, T] \times \mathbf{R}^{d} \ni(t, x) \mapsto \partial_{x} u^{\alpha, \varepsilon}(t, x) \sigma(t, x)$.

\section{Expansion of BSDE}

In this section, we show our result for the expansion of $\left(Y^{\alpha, \varepsilon}, Z^{\alpha, \varepsilon}\right)$ around $\alpha=0$. As for the $\varepsilon$-expansion around $\varepsilon=0$, we will discuss it in the following sections.

Firstly, in the case of $\alpha=0$ in $(8),\left(Y^{t, x, 0, \varepsilon}, Z^{t, x, 0, \varepsilon}\right)$ becomes the solution to the following linear BSDE:

$$
Y_{t}^{t, x, 0, \varepsilon}=g\left(X_{T}^{t, x, \varepsilon}\right)-\int_{t}^{T} Z_{s}^{t, x, 0, \varepsilon} \cdot d W_{s} .
$$

Then, we also have

$$
u^{0, \varepsilon}(t, x)=Y_{t}^{t, x, 0, \varepsilon}, \text { and } \partial_{x} u^{0}(t, x) \sigma(t, x)=Z_{t}^{t, x, 0, \varepsilon}
$$

\subsection{Notations and Basic Result}

For the preparation, we list up the notations and a lemma following Ma and Zhang (2002), which will be frequently used in the next subsection. Firstly, let $X$ denote a generic Banach space, and $\mathbf{E}\left(\right.$ or $\left.\mathbf{E}_{1}\right)$ denote a generic Euclidean space.

- $L^{0}([t, T] ; X):$ for $t \in[0, T]$, the space of all measurable functions $\varphi:[t, T] \mapsto X$.

- $C([t, T] ; X)$ : for $t \in[0, T]$, the space of all continuous functions $\varphi:[t, T] \mapsto X$; further for any $p>0$ $|\varphi|_{t, T}^{*, p}:=\sup _{t \leq s \leq T}\|\varphi\|_{X}^{p}$

- $C\left(\mathbf{F},[0, T] \times \mathbf{E} ; \mathbf{E}_{1}\right)$ : the space of all $\mathbf{E}_{1}$-valued, continuous random fields, $\varphi: \Omega \times[0, T] \times \mathbf{E} \mapsto \mathbf{E}_{1}$, such that for fixed $e \in \mathbf{E}, \varphi(\cdot, \cdot, e)$ is an $\mathbf{F}$-adapted process.

- $W^{1, \infty}\left(\mathbf{E} ; \mathbf{E}_{1}\right)$ : the space of all measurable functions $\psi: \mathbf{E} \mapsto \mathbf{E}_{1}$, such that for some constant $K>0$ it holds that

$$
\|\psi(x)-\psi(y)\|_{\mathbf{E}_{1}} \leq K\|x-y\|_{\mathbf{E}}, \forall x, y \in \mathbf{E} .
$$

- $L^{p}(\mathcal{G} ; \mathbf{E})$ : for any sub- $\sigma$-field $\mathcal{G} \subseteq \mathcal{F}_{T}$ and $0 \leq p<\infty$, the space of all E-valued, $\mathcal{G}$ - measurable random variables $\xi$ such that $E\left[|\xi|^{p}\right]<\infty$.

$L^{\infty}(\mathcal{G} ; \mathbf{E})$ : for any sub- $\sigma$-field $\mathcal{G} \subseteq \mathcal{F}_{T}$, the space of all $\mathbf{E}$-valued, $\mathcal{G}$ - measurable and bounded random variables. - $L^{p}(\mathbf{F},[0, T] ; X)$ : for $0 \leq p<\infty$, the space of all $X$-valued, $\mathbf{F}$-adapted processes $\xi$ such that $E\left[\int_{0}^{T}\left\|\xi_{t}\right\|_{X}^{p} d t\right]<$
$\infty$.

$L^{\infty}(\mathbf{F},[0, T] ; X)$ : the space of all $X$-valued, $\mathbf{F}$-adapted processes $\xi$ uniformly bounded in $(t, \omega)$.

Lemma 3.1 (Lemma 2.2. in Ma and Zhang (2002)) 
1. Suppose that $\tilde{b} \in C\left(\mathbf{F},[0, T] \times \mathbf{R}^{d} ; \mathbf{R}^{d}\right) \cap L^{0}\left([0, T] ; W^{1, \infty}\left(\mathbf{R}^{d} ; \mathbf{R}^{d}\right)\right), \tilde{\sigma} \in C\left(\mathbf{F},[0, T] \times \mathbf{R}^{d} ; \times \mathbf{R}^{d \times d}\right) \cap L^{0}\left([0, T] ; W^{1, \infty}\left(\mathbf{R}^{d} ; \mathbf{R}^{d \times d}\right)\right)$, with a common Lipschitz constant $K>0$. Suppose also that $\tilde{b}(t, 0)=0$ and $\tilde{\sigma}(t, 0)=0$ P-a.s. For any $h^{0} \in L^{2}\left(\mathbf{F},[0, T] ; \mathbf{R}^{d}\right)$ and $h^{1} \in L^{2}\left(\mathbf{F},[0, T] ; \mathbf{R}^{d \times d}\right)$, let $X$ be the solution of the following SDE:

$$
X_{t}=x+\int_{0}^{t}\left[\tilde{b}\left(s, X_{s}\right)+h_{s}^{0}\right] d s+\int_{0}^{t}\left[\tilde{\sigma}\left(s, X_{s}\right)+h_{s}^{1}\right] d W_{s}
$$

Then, for any $p \geq 2$, there exists a constant $C>0$ depending only on $p, T$ and $K$, such that

$$
E\left[|X|_{t, T}^{*, p}\right] \leq C\left\{|x|^{p}+E\left[\int_{0}^{T}\left[\left.|| h_{t}^{0}\right|^{p}+\left|h_{t}^{1}\right|^{p}\right] d t\right]\right\},
$$

where $|X|_{t, T}^{*, p}:=\sup _{t \leq s \leq T}\left\|X_{s}\right\|^{p}$.

2. Assume that $\tilde{f} \in C\left(\mathbf{F},[0, T] \times \mathbf{R} \times \mathbf{R}^{d} ; \mathbf{R}\right) \cap L^{0}\left([0, T] ; W^{1, \infty}\left(\mathbf{R} \times \mathbf{R}^{d}\right)\right)$ with a uniform Lipschitz constant $K>0$, and $\tilde{f}(\omega, s, 0,0)=0$ P-a.e. $\omega \in \Omega$. For any $\xi \in L^{2}\left(\mathcal{F}_{T} ; \mathbf{R}\right)$ and $h \in L^{2}(\mathbf{F},[0, T] ; \mathbf{R})$, let $(Y, Z)$ be the adapted solution to the BSDE:

$$
Y_{t}=\xi+\int_{t}^{T}\left[\tilde{f}\left(s, Y_{s}, Z_{s}\right)+h_{s}\right] d s-\int_{t}^{T} Z_{s} \cdot d W_{s} .
$$

Then there exists a constant $C>0$ depending only on $T$ and the Lipschitz constant of $\tilde{f}$, such that

$$
E\left[\int_{0}^{T}\left|Z_{t}\right|^{2} d t\right] \leq C E\left[|\xi|^{2}+\int_{0}^{T}\left|h_{t}\right|^{2} d t\right] .
$$

Moreover, for all $p \geq 2$, there exists a constant $C_{p}>0$, such that

$$
E\left[|Y|_{t, T}^{*, p}\right] \leq C_{p} E\left[|\xi|^{p}+\int_{0}^{T}\left|h_{t}\right|^{p} d t\right]
$$

where $|Y|_{t, T}^{*, p}:=\sup _{t \leq s \leq T}\left\|Y_{s}\right\|^{p}$.

Also, in order to estimate the expansion error we define a space as in Takahashi and Yamada (2012). For any $\beta, \mu>0$, let $H_{\beta, \mu, T}$ be the space of functions $v:[0, T] \times \mathbf{R}^{d} \rightarrow \mathbf{R}^{n}$ such that

$$
\|v\|_{H_{\beta, \mu, T}}^{2}=\int_{0}^{T} \int_{\mathbf{R}^{d}} e^{\beta s}|v(s, x)|^{2} e^{-\mu|x|} d x d s<\infty .
$$

\subsection{Asymptotic Expansion for BSDE and its Representation}

Hereafter, we often suppress the subscript $\varepsilon$ for the notational simplicity. Also we frequently use abbreviated notations such as $Y_{t}^{\alpha}, Z_{t}^{\alpha}, u^{\alpha}$ and $\partial_{x} u^{\alpha} \sigma$ in stead of $Y_{t}^{t, x, \alpha, \varepsilon}, Z_{t}^{t, x, \alpha, \varepsilon}, u^{\alpha, \varepsilon}$ and $\partial_{x} u^{\alpha, \varepsilon} \sigma$, respectively.

Moreover, we use the following notations and the abbreviations especially in the next theorem:

$$
\begin{aligned}
& \sum_{\mathbf{n}_{\beta}, \mathbf{d}^{(\beta)}}^{(n)}:=\sum_{\beta=1}^{n} \sum_{\mathbf{n}_{\beta} \in L_{n, \beta}} \sum_{\mathbf{d}^{(\beta)} \in\{1, \cdots, d+1\}^{\beta}} \frac{1}{\beta !}, \\
& \sum_{\mathbf{n}_{\beta}, \mathbf{d}^{(\beta)}, \beta=2}^{(n)}:=\sum_{\beta=2}^{n} \sum_{\mathbf{n}_{\beta} \in L_{n, \beta}} \sum_{\mathbf{d}^{(\beta)} \in\{1, \cdots, d+1\}^{\beta}} \frac{1}{\beta !}, \\
& L_{n, \beta}:=\left\{\mathbf{n}_{\beta}=\left(n_{1}, \cdots, n_{\beta}\right) ; \sum_{k=1}^{\beta} n_{k}=n ;\left(n, n_{k}, \beta \in \mathbf{N}\right)\right\}, \\
& Z^{t, x, \alpha, \varepsilon}=\left(Z^{t, x, \alpha, \varepsilon, 1}, \cdots, Z^{t, x, \alpha, \varepsilon, d}\right), \\
& \partial_{\alpha} Z^{\alpha} \equiv \partial_{\alpha} Z^{t, x, \alpha, \varepsilon}=\left(\partial_{\alpha} Z^{t, x, \alpha, \varepsilon, 1}, \cdots, \partial_{\alpha} Z^{t, x, \alpha, \varepsilon, d}\right), \\
& \Xi^{\alpha} \equiv \Xi^{t, x, \alpha, \varepsilon}:=\left(Y^{t, x, \alpha, \varepsilon}, Z^{t, x, \alpha, \varepsilon}\right), \\
& \Xi^{\alpha, d_{k}} \equiv \Xi^{t, x, \alpha, \varepsilon, d_{k}} \\
& \Theta_{r}^{\alpha} \equiv \Theta_{r}^{t, x, \alpha, \varepsilon}:=\left(r, X_{r}^{t, x, \varepsilon}, \Xi_{r}^{t, x, \alpha, \varepsilon}\right)=\left(r, X_{r}^{t, x, \varepsilon}, Y_{r}^{t, x, \alpha, \varepsilon}, Z^{t, x, \alpha, \varepsilon}\right), \\
& \partial_{\mathbf{d}^{(\beta)}} f\left(\Theta_{r}^{\alpha}\right):=\frac{\partial^{\beta}}{\partial \xi_{d_{1}} \cdots \partial \xi_{d_{\beta}}} f\left(\cdot, \cdot, \Xi_{r}^{t, x, \alpha, \varepsilon}\right)=\frac{\partial^{\beta}}{\partial \xi_{d_{1}} \cdots \partial \xi_{d_{\beta}}} f\left(\cdot, \cdot, Y_{r}^{t, x, \alpha, \varepsilon}, Z^{t, x, \alpha, \varepsilon}\right), \\
& \left(\mathbf{d}^{(\beta)}:=\left(d_{1}, \cdots, d_{\beta}\right) \in\{1, \cdots, d+1\}^{\beta}, \beta \geq 1\right), \\
& \partial_{y} f\left(\Theta_{r}^{\alpha}\right):=\frac{\partial}{\partial y} f\left(\cdot, \cdot, Y_{r}^{t, x, \alpha, \varepsilon}, \cdot\right) \text {, } \\
& \nabla_{z} f\left(\Theta_{r}^{\alpha}\right):=\left(\frac{\partial f\left(\cdot, \cdot, \cdot, Z_{r}^{t, x, \alpha, \varepsilon}\right)}{\partial z_{1}}, \cdots, \frac{\partial f\left(\cdot, \cdot, \cdot, Z_{r}^{t, x, \alpha, \varepsilon}\right)}{\partial z_{d}}\right) .
\end{aligned}
$$


Section 2.4 of El Karoui et al. (1997) discuss the first-order differentiation of the function $\alpha \mapsto\left(Y^{\alpha}, Z^{\alpha}\right)$. In the following theorem, we provide a representation of $\partial_{\alpha}^{n} Y_{s}^{t, x, \alpha}:=\frac{\partial^{n}}{\partial \alpha^{n}} Y_{s}^{t, x, \alpha}$ and $\partial_{\alpha}^{n} Z_{s}^{t, x, \alpha}:=\frac{\partial^{n}}{\partial \alpha^{n}} Z_{s}^{t, x, \alpha}$ for any $n \in \mathbf{N}$ and derive an asymptotic expansion of $\left(Y^{\alpha}, Z^{\alpha}\right)$ with respect to the parameter $\alpha$ around $\alpha=0$.

Theorem 3.1 Given the forward $S D E(7)$ and $Y^{t, x, 0}$ in (11), for $s \in[t, T]$, the derivatives $\partial_{\alpha}^{n} Y_{s}^{t, x, \alpha}=\frac{\partial^{n}}{\partial \alpha^{n}} Y_{s}^{t, x, \alpha}$ and $\partial_{\alpha}^{n} Z_{s}^{t, x, \alpha}=\frac{\partial^{n}}{\partial \alpha^{n}} Z_{s}^{t, x, \alpha}$ satisfy:

when $n=1$,

$$
\partial_{\alpha} Y_{s}^{t, x, \alpha}=\int_{s}^{T}\left[f\left(\Theta_{r}^{\alpha}\right)+\alpha \partial_{y} f\left(\Theta_{r}^{\alpha}\right)\left(\partial_{\alpha} Y_{r}^{\alpha}\right)+\alpha \nabla_{z} f\left(\Theta_{r}^{\alpha}\right) \cdot\left(\partial_{\alpha} Z_{r}^{\alpha}\right)\right] d r-\int_{s}^{T}\left(\partial_{\alpha} Z_{r}^{\alpha}\right) \cdot d W_{r}
$$

when $n \geq 2$,

$$
\partial_{\alpha}^{n} Y_{s}^{t, x, \alpha}=\int_{s}^{T}\left[H^{n}(r, t, x, \alpha)+\alpha\left\{\partial_{y} f\left(\Theta_{r}^{\alpha}\right) \partial_{\alpha}^{n} Y_{r}^{\alpha}+\nabla_{z} f\left(\Theta_{r}^{\alpha}\right) \cdot \partial_{\alpha}^{n} Z_{r}^{\alpha}\right\}\right] d r-\int_{s}^{T} \partial_{\alpha}^{n} Z_{r}^{\alpha} \cdot d W_{r},
$$

where

$$
H^{n}(r, t, x, \alpha):=n ! \sum_{\mathbf{n}_{\beta}, \mathbf{d}^{(\beta)}}^{(n-1)} \partial_{\mathbf{d}(\beta)} f\left(\Theta_{r}^{\alpha}\right) \prod_{k=1}^{\beta} \frac{1}{n_{k} !} \partial_{\alpha}^{n_{k}} \Xi^{\alpha, d_{k}}+\alpha n ! \sum_{\mathbf{n}_{\beta}, \mathbf{d}^{(\beta)}, \beta=2}^{(n)} \partial_{\mathbf{d}^{(\beta)}} f\left(\Theta_{r}^{\alpha}\right) \prod_{k=1}^{\beta} \frac{1}{n_{k} !} \partial_{\alpha}^{n_{k}} \Xi^{\alpha, d_{k}} .
$$

Moreover, for any $M \in \mathbf{N}$, there exists a constant $C(M, T)>0$ such that

$$
\left\|u^{\alpha, \varepsilon}-\left\{u^{0, \varepsilon}+\sum_{i=1}^{M} \alpha^{i} u_{i}^{0, \varepsilon}\right\}\right\|_{H_{\beta, \mu, T}}^{2}+\left\|\partial_{x} u^{\alpha, \varepsilon} \sigma-\left\{\partial_{x} u^{0, \varepsilon} \sigma+\sum_{i=1}^{M} \alpha^{i} \partial_{x} u_{i}^{0, \varepsilon} \sigma\right\}\right\|_{H_{\beta, \mu, T}}^{2} \leq \alpha^{2(M+1)} C(M, T),
$$

where

$$
\begin{aligned}
u^{0, \varepsilon}(t, x) & =Y_{t}^{t, x, 0, \varepsilon}=E\left[g\left(X_{T}^{t, x, \varepsilon}\right)\right] \\
\partial_{x} u^{0, \varepsilon} \sigma(t, x) & =Z_{t}^{t, x, 0, \varepsilon}=E\left[g\left(X_{T}^{t, x, \varepsilon}\right) N_{T}^{t, x, \varepsilon}\right] \sigma(t, x),
\end{aligned}
$$

and

$$
\begin{aligned}
u_{n+1}^{0, \varepsilon}(t, x) & =\left.\frac{1}{(n+1) !} \partial_{\alpha}^{n+1} Y_{t}^{t, x, \alpha, \varepsilon}\right|_{\alpha=0}=E\left[\int_{t}^{T} F^{n+1}\left(r, X_{r}^{t, x, \varepsilon}\right) d r\right], \text { for } n=0,1, \cdots, \\
\partial_{x} u_{n+1}^{0, \varepsilon} \sigma(t, x) & =\left.\frac{1}{(n+1) !} \partial_{\alpha}^{n+1} Z_{t}^{t, x, \alpha, \varepsilon}\right|_{\alpha=0}=E\left[\int_{t}^{T}\left[F^{n+1}\left(r, X_{r}^{t, x, \varepsilon}\right)\right] N_{r}^{t, x, \varepsilon} d r\right] \sigma(t, x), \text { for } n=0,1, \cdots
\end{aligned}
$$

where $N_{r}^{t, x, \varepsilon}$ stands for the Malliavin Delta weight:

$$
N_{r}^{t, x, \varepsilon}=\frac{1}{(r-t)} \int_{t}^{r} \sigma\left(X_{\tau}^{t, x, \varepsilon}\right)^{-1} \nabla X_{\tau}^{t, x, \varepsilon} d W_{\tau} .
$$

Here, $F^{n+1}, n \geq 0$, is recursively given by

$$
\begin{gathered}
F^{1}(t, x)=f\left(t, x, u^{0, \varepsilon}(t, x), \partial_{x} u^{0, \varepsilon} \sigma(t, x)\right), \text { for } n=0, \\
F^{n+1}(t, x)=\sum_{\mathbf{n}_{\beta}, \mathbf{d}^{(\beta)}}^{(n)} \partial_{\mathbf{d}^{(\beta)}} f\left(t, x, u^{0, \varepsilon}(t, x), \partial_{x} u^{0, \varepsilon} \sigma(t, x)\right) \prod_{k=1}^{\beta} \frac{1}{n_{k} !} \partial_{\alpha}^{n_{k}} \hat{\Xi}^{0, d_{k}}, \text { for } n \geq 1,
\end{gathered}
$$

where

$$
\begin{aligned}
& \left.\partial_{x} u^{0, \varepsilon} \sigma(t, x)=\left(\partial_{x} u^{0, \varepsilon} \sigma(t, x)\right)^{1}, \cdots,\left(\partial_{x} u^{0, \varepsilon} \sigma(t, x)\right)^{d}\right), \\
& \hat{\Xi}^{0} \equiv \hat{\Xi}^{t, x, 0, \varepsilon}:=\left(u^{0, \varepsilon}(t, x), \partial_{x} u^{0, \varepsilon} \sigma(t, x)\right)=\left(u^{0, \varepsilon}(t, x),\left(\partial_{x} u^{0, \varepsilon} \sigma(t, x)\right)^{1}, \cdots,\left(\partial_{x} u^{0, \varepsilon} \sigma(t, x)\right)^{d}\right), \\
& \hat{\Xi}^{0, d_{k}} \equiv \hat{\Xi}^{t, x, 0, \varepsilon, d_{k}} .
\end{aligned}
$$

Remark 3.1 In the case of $d=1$, (23) and (24) is reduced to the following equations:

$$
\begin{aligned}
d\left(\partial_{\alpha} Y_{r}^{\alpha}\right) & =-\left[f\left(\Theta_{r}^{\alpha}\right)+\alpha \partial_{y} f\left(\Theta_{r}^{\alpha}\right)\left(\partial_{\alpha} Y_{r}^{\alpha}\right)+\alpha \partial_{z} f\left(\Theta_{r}^{\alpha}\right)\left(\partial_{\alpha} Z_{r}^{\alpha}\right)\right] d r+\partial_{\alpha} Z_{r}^{\alpha} d W_{r}, \quad \text { for } n=1, \\
d\left(\partial_{\alpha}^{n} Y_{r}^{\alpha}\right) & =-\left[H^{n}(r, t, x, \alpha)+\alpha\left\{\partial_{y} f\left(\Theta_{r}^{\alpha}\right) \partial_{\alpha}^{n} Y_{r}^{\alpha}+\partial_{z} f\left(\Theta_{r}^{\alpha}\right) \partial_{\alpha}^{n} Z_{r}^{\alpha}\right\}\right] d r,+\partial_{\alpha}^{n} Z_{r}^{\alpha} d W_{r}, \quad \text { for } n \geq 2 \\
\partial_{\alpha}^{n} Y_{T}^{\alpha} & =0,
\end{aligned}
$$


where

$$
\begin{aligned}
& H^{n}(r, t, x, \alpha)=n ! \sum_{k=1}^{n-1} \sum_{\beta_{1}+\cdots+\beta_{k}=n-1, \beta_{i} \geq 1} \sum_{i=0}^{k} \frac{1}{i !(k-i) !} \partial_{y}^{k-i} \partial_{z}^{i} f\left(\Theta_{r}^{\alpha}\right) \prod_{j=1}^{k-i} \frac{1}{\beta_{j} !} \partial_{\alpha}^{\beta_{j}} Y_{r}^{\alpha} \prod_{j=k-i+1}^{k} \frac{1}{\beta_{j} !} \partial_{\alpha}^{\beta_{j}} Z_{r}^{\alpha} \\
& +\alpha n ! \sum_{k=2}^{n} \sum_{\beta_{1}+\cdots+\beta_{k}=n, \beta_{i} \geq 1} \sum_{i=0}^{k} \frac{1}{i !(k-i) !} \partial_{y}^{k-i} \partial_{z}^{i} f\left(\Theta_{r}^{\alpha}\right) \prod_{j=1}^{k-i} \frac{1}{\beta_{j} !} \partial_{\alpha}^{\beta_{j}} Y_{r}^{\alpha} \prod_{j=k-i+1}^{k} \frac{1}{\beta_{j} !} \partial_{\alpha}^{\beta_{j}} Z_{r}^{\alpha},
\end{aligned}
$$

and $\prod_{j}^{i} \equiv 1$ when $i<j$.

In addition, $F^{n+1}, n \geq 1$, is recursively given by

$$
\begin{aligned}
F^{1}(t, x)= & f\left(t, x, u^{0, \varepsilon}(t, x), \partial_{x} u^{0, \varepsilon} \sigma(t, x)\right), \text { for } n=0, \\
F^{n+1}(t, x)= & \sum_{k=1}^{n} \sum_{\beta_{1}+\cdots+\beta_{k}=n, \beta_{i} \geq 1} \sum_{i=0}^{k} \frac{1}{i !(k-i) !} \\
& \partial_{y}^{k-i} \partial_{z}^{i} f\left(t, x, u^{0, \varepsilon}(t, x), \partial_{x} u^{0, \varepsilon} \sigma(t, x)\right) \prod_{j=1}^{k-i} \frac{1}{\beta_{j} !} u_{\beta_{j}}^{0, \varepsilon}(t, x) \prod_{j=k-i+1}^{k} \frac{1}{\beta_{j} !} \partial_{x} u_{\beta_{j}}^{0, \varepsilon} \sigma(t, x), \text { for } n \geq 1 .
\end{aligned}
$$

\section{Proof.}

We only prove the case of $d=1$ for the notational simplicity.

Firstly, as in the beginning of this section, $\left(Y^{0}, Z^{0}\right)$ is the solution to linear BSDE:

$$
Y_{t}^{0}=g\left(X_{T}^{\varepsilon}\right)-\int_{t}^{T} Z_{s}^{0} d W_{s}
$$

We have

$$
u^{0}(t, x)=Y_{t}^{t, x, 0}
$$

and by Theorem 4.2 of Ma-Zhang (2002) with null driver,

$$
\partial_{x} u^{0}(t, x) \sigma(t, x)=Z_{t}^{t, x, 0}
$$

has the representation (28).

Next, we will apply an induction argument to the the number of the times of the differentiation of $\left(Y^{\alpha}, Z^{\alpha}\right)$ with respect to $\alpha$, and then will prove the expansion (26). We also remark that we will use a generic constant $C>0$, which is allowed to vary, depending on some constants associated with Assumption 2.1, Lemma 3.1, the time horizon the number of the times of the differentiation and so on.

- $n=1\left(\partial_{\alpha} Y_{t}^{\alpha}\right)$

In the first place, let us show the case of the first order differentiation with respect to $\alpha$. For an arbitrary initial condition $(t, x) \in[0, T] \times \mathbf{R}^{d}$, let $\left(Y_{1, s}^{t, x, \alpha}, Z_{1, s}^{t, x, \alpha}\right)_{t \leq s \leq T}$ be the solution to the BSDE, which is obtained by the formal differentiation of (8) with respect to $\alpha$ :

$$
\begin{aligned}
Y_{1, s}^{t, x, \alpha}= & \int_{s}^{T}\left[f\left(\Theta_{r}^{t, x, \alpha}\right)+\alpha \partial_{y} f\left(\Theta_{r}^{t, x, \alpha}\right) Y_{1, r}^{t, x, \alpha}+\alpha \partial_{z} f\left(\Theta_{r}^{t, x, \alpha}\right) Z_{1, r}^{t, x, \alpha}\right] d r \\
& -\int_{s}^{T} Z_{1, r}^{t, x, \alpha} d W_{r}
\end{aligned}
$$

Applying Proposition 2.4 with its remark in p.29 of El Karoui et al. (1997) or the similar argument as in the proof of Theorem 3.1 in Ma and Zhang (2002), we can see $\left(Y_{1, s}^{t, x, \alpha}, Z_{1, s}^{t, x, \alpha}\right)_{t \leq s \leq T}$ satisfies:

$$
\lim _{h \rightarrow 0} E\left[\sup _{t \leq s \leq T}\left|\frac{Y_{s}^{t, x, \alpha+h}-Y_{s}^{t, x, \alpha}}{h}-Y_{1, s}^{t, x, \alpha}\right|^{2}+\sup _{t \leq s \leq T}\left|Y_{s}^{t, x, \alpha+h}-Y_{s}^{t, x, \alpha}\right|^{2}\right]=0
$$

and

$$
\lim _{h \rightarrow 0} E\left[\int_{t}^{T}\left|\frac{Z_{s}^{t, x, \alpha+h}-Z_{s}^{t, x, \alpha}}{h}-Z_{1, s}^{t, x, \alpha}\right|^{2} d s+\int_{t}^{T}\left|Z_{s}^{t, x, \alpha+h}-Z_{s}^{t, x, \alpha}\right|^{2} d s\right]=0 .
$$

Hence, hereafter we often write $Y_{1, s}^{t, x, \alpha}$ for $\partial_{\alpha} Y_{s}^{t, x, \alpha}$ and $Z_{1, s}^{t, x, \alpha}$ for $\partial_{\alpha} Z_{s}^{t, x, \alpha}$. 
Next, define

$$
u_{1}^{\alpha}(t, x):=E\left[\int_{t}^{T}\left[f\left(\Theta_{r}^{t, x, \alpha}\right)+\alpha \partial_{y} f\left(\Theta_{r}^{t, x}\right) Y_{1, r}^{t, x, \alpha}+\alpha \partial_{z} f\left(\Theta_{r}^{t, x, \alpha}\right) Z_{1, r}^{t, x, \alpha}\right] d r\right]
$$

and

$$
v_{1}^{\alpha}(t, x):=\frac{1}{\varepsilon} E\left[\int_{t}^{T}\left[f\left(\Theta_{r}^{t, x, \alpha}\right)+\alpha \partial_{y} f\left(\Theta_{r}^{t, x}\right) Y_{1, r}^{t, x, \alpha}+\alpha \partial_{z} f\left(\Theta_{r}^{t, x, \alpha}\right) Z_{1, r}^{t, x, \alpha}\right] N_{r}^{t, x} d r\right],
$$

where $\left(N_{r}^{t, x}\right)_{t \leq r \leq T}$ is the Malliavin delta weight given by (31). First, it holds that $u_{1}^{\alpha}(t, x)=\partial_{\alpha} Y_{t}^{t, x, \alpha}$. Second, since $f, \partial_{y} f$ and $\partial_{z} f$ are bounded by Assumption 2-1-3., and Lemma 3.1-2. is applied to (43), there exists $C_{1}$ such that for all $p>0$,

$$
E\left[\left|Y_{1}^{t, x, \alpha}\right|_{t, T}^{*, p}+\int_{t}^{T}\left|Z_{1, r}^{t, x, \alpha}\right|^{2} d r\right] \leq C_{1}
$$

which is applied to (46) to obtain $\left|u_{1}^{\alpha}(t, x)\right| \leq C$ for some constant $C$ for all $(t, x)$.

Next we consider the solution to the variational equation of the BSDE (??):

$$
\nabla Y_{1, s}^{t, x, \alpha}=\int_{s}^{T}\left[B^{1}(r, t, x, \alpha)+\alpha \partial_{y} f\left(\Theta_{r}^{t, x, \alpha}\right) \nabla Y_{1, r}^{t, x, \alpha}+\alpha \partial_{z} f\left(\Theta_{r}^{t, x, \alpha}\right) \nabla Z_{1, r}^{t, x, \alpha}\right] d r-\int_{s}^{T} \nabla Z_{1, r}^{t, x, \alpha} d W_{r},
$$

where

$$
\begin{aligned}
B^{1}(r, t, x, \alpha)= & \partial_{x} f\left(\Theta_{r}^{t, x, \alpha}\right) \nabla X_{r}^{t, x}+\partial_{y} f\left(\Theta_{r}^{t, x, \alpha}\right) \nabla Y_{r}^{t, x, \alpha}+\partial_{z} f\left(\Theta_{r}^{t, x, \alpha}\right) \nabla Z_{r}^{t, x, \alpha} \\
& +\alpha \partial_{x y} f\left(\Theta_{r}^{t, x}\right) \nabla X_{r}^{t, x} Y_{1, r}^{t, x, \alpha}+\alpha \partial_{y^{2}} f\left(\Theta_{r}^{t, x}\right) \nabla Y_{r}^{t, x, \alpha} Y_{1, r}^{t, x, \alpha}+\alpha \partial_{y z} f\left(\Theta_{r}^{t, x}\right) \nabla Z_{r}^{t, x} Y_{1, r}^{t, x, \alpha} \\
& +\alpha \partial_{x z} f\left(\Theta_{r}^{t, x}\right) \nabla X_{r}^{t, x} Z_{1, r}^{t, x, \alpha}+\alpha \partial_{y z} f\left(\Theta_{r}^{t, x}\right) \nabla Y_{r}^{t, x, \alpha} Z_{1, r}^{t, x, \alpha}+\alpha \partial_{z^{2}} f\left(\Theta_{r}^{t, x}\right) \nabla Z_{r}^{t, x} Z_{1, r}^{t, x, \alpha} .
\end{aligned}
$$

First, note that due to Lemma 3.1, we have for all $p>0$,

$$
E\left[\left|\nabla X^{t, x}\right|_{t, T}^{*, p}+\left|\nabla Y^{t, x, \alpha}\right|_{t, T}^{*, p}\right] \leq C_{2} \text { for some constant } C_{2}
$$

By Theorem 3.1-(iii) in Ma and Zhang (2002) we also know that:

$$
Z_{s}^{t, x}=\partial_{x} u\left(s, X_{s}^{s, x}\right) \sigma\left(s, X_{s}^{s, x}\right), \forall s \in[t, T], P-a . s .
$$

Thus, we have

$$
\nabla_{x} Z_{s}^{t, x}=\partial_{x}^{2} u\left(s, X_{s}^{s, x}\right) \nabla X_{s}^{t, x} \sigma\left(s, X_{s}^{s, x}\right)+\partial_{x} u\left(s, X_{s}^{s, x}\right) \partial_{x} \sigma\left(s, X_{s}^{s, x}\right) \nabla X_{s}^{t, x}, \forall s \in[t, T], P-a . s .
$$

Moreover, by Lemma 3.4. of Crisan and Delarue (2012), $\partial_{x} u$ and $\partial_{x}^{2} u$ are bounded. Hence with Assumption 2.1.1 and (51) we obtain for all $p>0$,

$$
E\left[\left|\nabla Z^{t, x}\right|_{t, T}^{*, p}\right] \leq C_{3} \text { for some constant } C_{3} .
$$

Then, applying Assumption 2.1-.3, (48), (51) and (54), we obtain

$$
E\left[\int_{t}^{T}\left|B^{1}(r, t, x, \alpha)\right|^{2} d r\right] \leq C_{4} \text {. for some constant } C_{4}
$$

Here, for instance, we use the following estimate: as for the last term in $B^{1}(r, t, x, \alpha)$ in (50), by the boundedness of $\partial_{z^{2}} f\left(\Theta_{r}^{t, x}\right)$ and the Hölder inequality with (48) and (54), we have for some constants $\hat{C}$ and $\bar{C}$ :

$$
\begin{aligned}
& \left.\left.\left.E\left[\int_{t}^{T} \mid \alpha \partial_{z^{2}} f\left(\Theta_{r}^{t, x}\right) \nabla Z_{r}^{t, x} Z_{1, r}^{t, x, \alpha}\right)\right|^{2}\right] \leq\left.\hat{C} E\left[\int_{t}^{T} \mid \nabla Z_{r}^{t, x} Z_{1, r}^{t, x, \alpha}\right)\right|^{2} d r\right] \\
& \left.\left.\leq\left.\hat{C} E\left[\left|\nabla Z^{t, x}\right|_{t, T}^{*, 2} \int_{t}^{T} \mid Z_{1, r}^{t, x, \alpha}\right)\right|^{2} d r\right] \leq \hat{C} E\left[\left(\left|\nabla Z^{t, x}\right|_{t, T}^{*, 2}\right)^{2}\right]^{1 / 2} E\left[\left.\left(\int_{t}^{T} \mid Z_{1, r}^{t, x, \alpha}\right)\right|^{2} d r\right)^{2}\right]^{1 / 2} \leq \bar{C} .
\end{aligned}
$$

Thus, applying Lemma 3.1 and the similar argument as in the proof of Theorem 3.1 in Ma and Zhang (2002) to (49), we have

$$
\lim _{h \rightarrow 0} E\left[\sup _{t \leq s \leq T}\left|\frac{Y_{1, s}^{t, x+h, \alpha}-Y_{1, s}^{t, x, \alpha}}{h}-\nabla Y_{1, s}^{t, x, \alpha}\right|^{2}+\sup _{t \leq s \leq T}\left|Y_{1, s}^{t, x+h, \alpha}-Y_{1, s}^{t, x, \alpha}\right|^{2}\right]=0,
$$




$$
\lim _{h \rightarrow 0} E\left[\int_{t}^{T}\left|\frac{Z_{1, s}^{t, x+h, \alpha}-Z_{1, s}^{t, x, \alpha}}{h}-\nabla Z_{1, s}^{t, x, \alpha}\right|^{2} d s+\int_{t}^{T}\left|Z_{1, s}^{t, x+h, \alpha}-Z_{1, s}^{t, x, \alpha}\right|^{2} d s\right]=0
$$

and

$$
E\left[\left|\nabla Y_{1}^{t, x, \alpha}\right|_{t, T}^{*, 2}+\int_{t}^{T}\left|\nabla Z_{1, r}^{t, x, \alpha}\right|^{2} d r\right] \leq \int_{t}^{T}\left|B^{1}(r, t, x, \alpha)\right|^{2} d r \leq C_{4} .
$$

Next, let

$$
\bar{v}_{1}^{\alpha}(t, x):=E\left[\int_{t}^{T}\left[B^{1}(r, t, x, \alpha)+\alpha \partial_{y} f\left(\Theta_{r}^{t, x, \alpha}\right) \nabla Y_{1, r}^{t, x, \alpha}+\alpha \partial_{z} f\left(\Theta_{r}^{t, x, \alpha}\right) \nabla Z_{1, r}^{t, x, \alpha}\right] d r\right] .
$$

Then, by Assumption 2-1-3., (55) and (59), we obtain $\left|\bar{v}_{1}^{\alpha}(t, x)\right| \leq C$.

Moreover, let us show $v_{1}^{\alpha}=\bar{v}_{1}^{\alpha}=\partial_{x} u_{1}^{\alpha}$ in the following way.

Firstly, using basic results of Malliavin calculus, we calculate the Malliavin derivatives of $f\left(r, \Theta_{r}\right),\left\{\partial_{y} f\left(\Theta_{r}^{t, x}\right) Y_{1, r}^{t, x, \alpha}\right\}$ and $\left\{\partial_{z} f\left(\Theta_{r}^{t, x}\right) Z_{1, r}^{t, x, \alpha}\right\}$ :

$$
\begin{aligned}
& D_{\tau}\left\{f\left(r, \Theta_{r}\right)\right\}=\left\{\partial_{x} f\left(\Theta_{r}^{t, x, \alpha}\right) \nabla X_{r}^{t, x}\right. \\
&+\partial_{y} f\left(\Theta_{r}^{t, x, \alpha}\right) \nabla Y_{r}^{t, x, \alpha} \\
&\left.+\partial_{z} f\left(\Theta_{r}^{t, x, \alpha}\right) \nabla Z_{r}^{t, x, \alpha}\right\}\left(\nabla X_{\tau}^{t, x}\right)^{-1} \varepsilon \sigma\left(\tau, X_{\tau}^{t, x}\right), \\
&=\quad\left\{D_{\tau} \partial_{y} f\left(\Theta_{r}^{t, x}\right)\right\} Y_{1, r}^{t, x, \alpha}+\partial_{y} f\left(\Theta_{r}^{t, x}\right)\left\{D_{\tau} Y_{1, r}^{t, x, \alpha}\right\} \\
&=\quad\left[\partial_{x y} f\left(\Theta_{r}^{t, x}\right) \nabla X_{r}^{t, x} Y_{1, r}^{t, x, \alpha}+\partial_{y^{2}} f\left(\Theta_{r}^{t, x}\right) \nabla Y_{r}^{t, x, \alpha} Y_{1, r}^{t, x, \alpha}+\partial_{y z} f\left(\Theta_{r}^{t, x}\right) \nabla Z_{r}^{t, x} Y_{1, r}^{t, x, \alpha}\right. \\
&\left.+\partial_{y} f\left(\Theta_{r}^{t, x}\right) \nabla Y_{1, r}^{t, x, \alpha}\right]\left(\nabla X_{\tau}^{t, x}\right)^{-1} \varepsilon \sigma\left(\tau, X_{\tau}^{t, x}\right), \\
& D_{\tau}\left\{\partial_{z} f\left(\Theta_{r}^{t, x}\right) Z_{1, r}^{t, x, \alpha}\right\} \quad \\
&=\left\{D_{\tau} \partial_{z} f\left(\Theta_{r}^{t, x}\right)\right\} Z_{1, r}^{t, x, \alpha}+\partial_{z} f\left(\Theta_{r}^{t, x}\right)\left\{D_{\tau} Z_{1, r}^{t, x, \alpha}\right\} \\
&=\quad\left[\partial_{x z} f\left(\Theta_{r}^{t, x}\right) \nabla X_{r}^{t, x} Z_{1, r}^{t, x, \alpha}+\partial_{y z} f\left(\Theta_{r}^{t, x}\right) \nabla Y_{r}^{t, x, \alpha} Z_{1, r}^{t, x, \alpha}+\partial_{z^{2}} f\left(\Theta_{r}^{t, x}\right) \nabla Z_{r}^{t, x} Z_{1, r}^{t, x, \alpha}\right. \\
&+\left.\partial_{z} f\left(\Theta_{r}^{t, x}\right) \nabla Z_{1, r}^{t, x, \alpha}\right]\left(\nabla X_{\tau}^{t, x}\right)^{-1} \varepsilon \sigma\left(\tau, X_{\tau}^{t, x}\right) .
\end{aligned}
$$

Then, by applying the integration by parts on the Wiener space, we have

$$
\begin{aligned}
& E\left[B^{1}(r, t, x, \alpha)+\alpha \partial_{y} f\left(\Theta_{r}^{t, x, \alpha}\right) \nabla Y_{1, r}^{t, x, \alpha}+\alpha \partial_{z} f\left(\Theta_{r}^{t, x, \alpha}\right) \nabla Z_{1, r}^{t, x, \alpha}\right] \\
= & E\left[\frac{1}{\varepsilon(r-t)} \int_{t}^{r} D_{\tau}\left\{f\left(\Theta_{r}^{t, x, \alpha}\right)+\alpha \partial_{y} f\left(\Theta_{r}^{t, x}\right) Y_{1, r}^{t, x, \alpha}+\alpha \partial_{z} f\left(\Theta_{r}^{t, x, \alpha}\right) Z_{1, r}^{t, x, \alpha}\right\} \sigma\left(\tau, X_{\tau}^{t, x}\right)^{-1}\left(\nabla X_{\tau}^{t, x}\right) d \tau\right] \\
= & \frac{1}{\varepsilon} E\left[\left\{f\left(\Theta_{r}^{t, x, \alpha}\right)+\alpha \partial_{y} f\left(\Theta_{r}^{t, x}\right) Y_{1, r}^{t, x, \alpha}+\alpha \partial_{z} f\left(\Theta_{r}^{t, x, \alpha}\right) Z_{1, r}^{t, x, \alpha}\right\} N_{r}^{t, x, \varepsilon}\right],
\end{aligned}
$$

where $N_{r}^{t, x, \varepsilon}$ is given by (31). Thus, we have $v_{1}^{\alpha}=\bar{v}_{1}^{\alpha}$, that is $(47)=(60)$.

Further, as $\partial_{x} u_{1}^{\alpha}(t, x)=\nabla Y_{1, t}^{t, x, \alpha}=\bar{v}_{1}^{\alpha}(t, x)$, we obtain that $v_{1}^{\alpha}=\bar{v}_{1}^{\alpha}=\partial_{x} u_{1}^{\alpha}$. Therefore, we conclude that for all $(t, x) \in[0, T] \times \mathbf{R}^{d}$,

$$
\left|\partial_{x} u_{1}^{\alpha}(t, x)\right| \leq C
$$

Moreover, following the similar argument of Theorem 3.1-(iii) of Ma and Zhang (2002), we know that

$$
Z_{1, s}^{t, x}=\partial_{x} u_{1}\left(s, X_{s}^{t, x}\right) \sigma\left(s, X_{s}^{t, x}\right) \forall s \in[t, T], P-a . s .
$$

Thus, with (61) and Assumption 2.1-1., we also have for all $p>0$,

$$
E\left[\left|Z_{1}^{t, x}\right|_{t, T}^{*, p}\right] \leq C
$$

- Induction

Based on the inductive argument, for an arbitrary fixed $n \in \mathbf{N}$ we assume that $\left(Y_{n, s}^{t, x, \alpha}, Z_{n, s}^{t, x, \alpha}\right)_{t \leq s \leq T}$ is the solution to the following BSDE:

$$
\begin{aligned}
Y_{n, s}^{t, x, \alpha}= & \int_{s}^{T}\left[H^{n}(r, t, x, \alpha)+\alpha \partial_{y} f\left(\Theta_{r}^{t, x, \alpha}\right) Y_{n, r}^{t, x, \alpha}+\alpha \partial_{z} f\left(\Theta_{r}^{t, x, \alpha}\right) Z_{n, r}^{t, x, \alpha}\right] d r \\
& -\int_{s}^{T} Z_{n, r}^{t, x, \alpha} d W_{r}
\end{aligned}
$$


where

$$
\begin{aligned}
H^{n}(r, t, x, \alpha)= & n ! \sum_{k=1}^{n-1} \sum_{\beta_{1}+\cdots+\beta_{k}=n-1, \beta_{l} \geq 1} \sum_{i=0}^{k} \frac{1}{i !(k-i) !} \partial_{y}^{k-i} \partial_{z}^{i} f\left(\Theta_{r}^{t, x, \alpha}\right) \prod_{j=1}^{k-i} \frac{1}{\beta_{j} !} Y_{\beta_{j}, r}^{t, x} \prod_{j=k-i+1}^{k} \frac{1}{\beta_{j} !} Z_{\beta_{j}, r}^{t, x, \alpha} \\
& +\alpha n ! \sum_{k=2}^{n} \sum_{\beta_{1}+\cdots+\beta_{k}=n, \beta_{l} \geq 1} \sum_{i=0}^{k} \frac{1}{i !(k-i) !} \partial_{y}^{k-i} \partial_{z}^{i} f\left(\Theta_{r}^{t, x, \alpha}\right) \prod_{j=1}^{k-i} \frac{1}{\beta_{j} !} Y_{\beta_{j}, r}^{t, x, \alpha} \prod_{j=k-i+1}^{k} \frac{1}{\beta_{j} !} Z_{\beta_{j}, r}^{t, x, \alpha}
\end{aligned}
$$

Here, for some constants $\bar{C}_{n}$ and $C_{n}$,

$$
E\left[\int_{t}^{T}\left|H^{n}(r, t, x, \alpha, \omega)\right|^{2} d r\right] \leq \bar{C}_{n}, E\left[\left|Y_{n}^{t, x, \alpha}\right|_{t, T}^{*, p}+\int_{t}^{T}\left|Z_{n, r}^{t, x, \alpha}\right|^{2} d r\right] \leq C_{n},
$$

and we also suppose that for all $p>0$,

$$
E\left[\left|\nabla Y_{n-1}^{t, x, \alpha}\right|_{t, T}^{*, p}+\left|\nabla Z_{n-1}^{t, x, \alpha}\right|_{t, T}^{*, p}\right] \leq \hat{C}_{n}, \text { for some constant } \hat{C}_{n} .
$$

Consequently, we assume that $u_{n}^{\alpha}(t, x)=\frac{1}{n !} \partial_{\alpha}^{n} Y_{t}^{t, x}$ and $Z_{n}^{t, x, \alpha}$ satisfy

$$
\left|u_{n}^{\alpha}(t, x)\right| \leq C,\left|\partial_{x} u_{n}^{\alpha}(t, x)\right| \leq C, E\left[\left|Z_{n}^{t, x, \alpha}\right|_{t, T}^{*, p}\right] \leq C, \forall p>0 .
$$

Let $\left(Y_{(n+1), s}^{t, x, \alpha}, Z_{(n+1), s}^{t, x, \alpha}\right)_{t \leq s \leq T}$ be the solution to the following BSDE which corresponds to the formal differentiation of the $\operatorname{BSDE}(63)$ with respect to $\alpha$ :

$$
\begin{aligned}
Y_{(n+1), s}^{t, x, \alpha}= & \int_{s}^{T}\left[H^{n+1}(r, t, x, \alpha)+\alpha \partial_{y} f\left(\Theta_{r}^{t, x, \alpha}\right) Y_{(n+1), r}^{t, x, \alpha}+f\left(\Theta_{r}^{t, x, \alpha}\right) \partial_{\alpha}^{n+1} Z_{(n+1), r}^{t, x, \alpha}\right] d r \\
& -\int_{s}^{T} Z_{(n+1), r}^{t, x, \alpha} d W_{r}
\end{aligned}
$$

where

$$
\begin{aligned}
& H^{n+1}(r, t, x, \alpha) \\
= & \partial_{\alpha} H^{n}(r, t, x, \alpha)+\partial_{y} f\left(\Theta_{r}^{t, x, \alpha}\right) Y_{n, r}^{t, x, \alpha}+\partial_{z} f\left(\Theta_{r}^{t, x, \alpha}\right) Z_{n, r}^{t, x, \alpha} \\
& +\alpha\left\{\partial_{\alpha} \partial_{y} f\left(\Theta_{r}^{t, x, \alpha}\right)\right\} Y_{n, r}^{t, x, \alpha}+\alpha\left\{\partial_{\alpha} \partial_{z} f\left(\Theta_{r}^{t, x, \alpha}\right)\right\} Z_{n, r}^{t, x, \alpha} \\
= & (n+1) ! \sum_{k=1}^{n} \sum_{\beta_{1}+\cdots+\beta_{k}=n, \beta_{i} \geq 1} \sum_{i=0}^{k} \frac{1}{i !(k-i) !} \partial_{y}^{k-i} \partial_{z}^{i} f\left(\Theta_{r}^{t, x, \alpha}\right) \prod_{j=1}^{k-i} \frac{1}{\beta_{j} !} Y_{\beta_{j}, r}^{t, x} \prod_{j=k-i+1}^{k} \frac{1}{\beta_{j} !} Z_{\beta_{j}, r}^{t, x, \alpha} \\
& +\alpha(n+1) ! \sum_{k=2}^{n+1} \sum_{\beta_{1}+\cdots+\beta_{k}=n+1, \beta_{i} \geq 1} \sum_{i=0}^{k} \frac{1}{i !(k-i) !} \partial_{y}^{k-i} \partial_{z}^{i} f\left(\Theta_{r}^{t, x, \alpha}\right) \prod_{j=1}^{k-i} \frac{1}{\beta_{j} !} Y_{\beta_{j}, r}^{t, x, \alpha} \prod_{j=k-i+1}^{k} \frac{1}{\beta_{j} !} Z_{\beta_{j}, r}^{t, x, \alpha} .
\end{aligned}
$$

Then, as in the case of $n=1$, following the similar argument as in the proof of Proposition 2.4 with its remark in p.29 of El Karoui et al. (1997) or in the proof of Theorem 3.1 of Ma and Zhang (2002), we are able to show

$$
\lim _{h \rightarrow 0} E\left[\sup _{t \leq s \leq T}\left|\frac{Y_{n, s}^{t, x, \alpha+h}-Y_{n, s}^{t, x, \alpha}}{h}-Y_{(n+1), s}^{t, x, \alpha}\right|^{2}+\sup _{t \leq s \leq T}\left|Y_{n, s}^{t, x, \alpha+h}-Y_{n, s}^{t, x, \alpha}\right|^{2}\right]=0,
$$

and

$$
\lim _{h \rightarrow 0} E\left[\int_{t}^{T}\left|\frac{Z_{n, s}^{t, x, \alpha+h}-Z_{n, s}^{t, x, \alpha}}{h}-Z_{(n+1), s}^{t, x, \alpha}\right|^{2} d s+\int_{t}^{T}\left|Z_{n, s}^{t, x, \alpha+h}-Z_{n, s}^{t, x, \alpha}\right|^{2} d s\right]=0 .
$$

Next, let

$$
u_{n+1}^{\alpha}(t, x)=\frac{1}{(n+1) !} E\left[\int_{t}^{T}\left[H^{n+1}(r, t, x, \alpha, \omega)+\alpha \partial_{y} f\left(\Theta_{r}^{t, x}\right) Y_{(n+1), r}^{t, x, \alpha}+\alpha \partial_{z} f\left(\Theta_{r}^{t, x, \alpha}\right) Z_{(n+1), r}^{t, x, \alpha}\right] d r\right] .
$$

Then, by using Assumption 2.1-3., (65) and (67) to apply Lemma 3-2. to (68), we have for some constants $\bar{C}_{n+1}$ and $C_{n+1}$,

$$
\begin{aligned}
& E\left[\int_{t}^{T}\left|H^{n+1}(r, t, x, \alpha, \omega)\right|^{2} d r\right] \leq \bar{C}_{n+1}, \\
& E\left[\left|Y_{(n+1)}^{t, x, \alpha}\right|_{t, T}^{*, p}+\int_{t}^{T}\left|Z_{(n+1), r}^{t, x, \alpha}\right|^{2} d r\right] \leq C_{n+1}, \text { for all } p>0
\end{aligned}
$$


and hence $\left|u_{n+1}^{\alpha}(t, x)\right| \leq C$.

Moreover, let

$$
v_{n+1}^{\alpha}(t, x)=\frac{1}{(n+1) !} \frac{1}{\varepsilon} E\left[\int_{t}^{T}\left[H^{n+1}(r, t, x, \alpha)+\alpha \partial_{y} f\left(\Theta_{r}^{t, x}\right) Y_{(n+1), r}^{t, x, \alpha}+\alpha \partial_{z} f\left(\Theta_{r}^{t, x, \alpha}\right) Z_{(n+1), r}^{t, x, \alpha}\right] N_{r}^{t, x} d r\right],
$$

where $\left(N_{r}^{t, x}\right)_{t \leq r \leq T}$ is the Malliavin Delta weight given by (31), again.

Then, as in the case $n=1, \partial_{x} u_{n+1}^{\alpha}(t, x)=\nabla Y_{(n+1), t}^{t, x, \alpha}$, and applying integration by parts on the Wiener space, we have $v_{n+1}^{\alpha}(t, x)=\partial_{x} u_{n+1}^{\alpha}(t, x)$ and $\left|\partial_{x} u_{n+1}^{\alpha}(t, x)\right| \leq C$.

- Asymptotic expansion (26):

By the Taylor expansion, we have the following formulas:

$$
\begin{gathered}
Y_{t}^{t, x, \alpha}=Y_{t}^{t, x, 0}+\left.\sum_{i=1}^{M} \frac{\alpha^{i}}{i !} \frac{\partial^{i}}{\partial \alpha^{i}} Y_{t}^{t, x, \alpha}\right|_{\alpha=0}+\left.\alpha^{M+1} \int_{0}^{1} \frac{(1-u)^{M}}{M !} \frac{\partial^{M+1}}{\partial \nu^{M+1}} Y_{t}^{t, x, \nu}\right|_{\nu=\alpha u} d u \\
=u^{0}(t, x)+\sum_{i=1}^{M} \alpha^{i} u_{i}^{0}(t, x)+\alpha^{M+1} \int_{0}^{1}(1-u)^{M} \tilde{u}_{M+1}^{\alpha u}(t, x) d u \\
Z_{t}^{t, x, \alpha}=Z_{t}^{, t, x, 0}+\left.\sum_{i=1}^{M} \frac{\alpha^{i}}{i !} \frac{\partial^{i}}{\partial \alpha^{i}} Z_{t}^{t, x, \alpha}\right|_{\alpha=0}+\left.\alpha^{M+1} \int_{0}^{1} \frac{(1-u)^{M}}{M !} \frac{\partial^{M+1}}{\partial \nu^{M+1}} Z_{t}^{t, x, \nu}\right|_{\nu=\alpha u} d u \\
=\partial_{x} u^{\alpha} \sigma(t, x)=\partial_{x} u^{0} \sigma(t, x)+\sum_{i=1}^{M} \alpha^{i} \partial_{x} u_{i}^{0} \sigma(t, x)+\alpha^{M+1} \int_{0}^{1}(1-u)^{M} \partial_{x} \tilde{u}_{M+1}^{\alpha u} \sigma(t, x) d u
\end{gathered}
$$

where $\tilde{u}_{M+1}^{\alpha}(t, x):=(M+1) u_{M+1}^{\alpha}(t, x)$ and $\partial_{x} \tilde{u}_{M+1}^{\alpha} \sigma(t, x):=(M+1) \partial_{x} u_{M+1}^{\alpha} \sigma(t, x)$.

On the other hand, by the previous result, we have $\left|\tilde{u}_{M+1}^{\alpha}(t, x)\right| \leq C$ and $\left|\partial_{x} \tilde{u}_{M+1}^{\alpha}(t, x)\right| \leq C$ for all $(t, x) \in$ $[0, T] \times \mathbf{R}^{d}$. Therefore, we finally obtain:

$$
\left\|u^{\alpha}-\left\{u^{0}+\sum_{i=1}^{M} \alpha^{i} u_{i}^{0}\right\}\right\|_{H_{\beta, \mu, T}}^{2}+\left\|\partial_{x} u^{\alpha} \sigma-\left\{\partial_{x} u^{0} \sigma+\sum_{i=1}^{M} \alpha^{i} \partial_{x} u_{i}^{0} \sigma\right\}\right\|_{H_{\beta, \mu, T}}^{2} \leq \alpha^{2(M+1)} C(M, T) .
$$

Then, we have the assertion.

\section{Expansion of FSDE}

Before providing our main result, we state an asymptotic expansion of $E\left[\varphi\left(X_{T}^{t, x, \varepsilon}\right)\right]$ in terms of a small diffusion parameter $\varepsilon$, which is a slight modification of Takahashi and Yamada (2013a, b). Here, $\varphi \in C_{b}^{\infty}, X_{T}^{t, x, \varepsilon}=$ $\left(X_{T}^{t, x, \varepsilon, 1}, \cdots, X_{T}^{t, x, \varepsilon, d}\right)$, and $X_{T}^{t, x, \varepsilon, i}, i=1, \cdots, d$ is the solution to the forward SDE (7) with $s=T$.

Firstly, let us present the Kusuoka-Stroock Functions, which is useful to clarify the order of a Wiener functional with respect to the time parameter $t$ in a unified manner, and thus to evaluate the error terms in asymptotic expansions.

\subsection{The Kusuoka-Stroock Functions}

This subsection introduces the space of Wiener functionals $\mathcal{K}_{r}^{T}$ developed by Kusuoka (2003) and its properties. The element of $\mathcal{K}_{r}^{T}$ is called the Kusuoka-Stroock function. See Nee (2010, 2011), Crisan and Delarue (2013) and Crisan et al. (2013) for more details of the notations and the proofs. Let $E$ be a separable Hilbert space and $\mathbf{D}^{n, \infty}(E)$ be the space of $E$-valued functionals that admit the Malliavin derivatives up to the $n$-th order. The following definition and lemma correspond to Definition 2.1 and Lemma 2.2 of Crisan and Delarue (2013).

Definition 4.1 Given $r \in \mathbf{R}$ and $n \in \mathbf{N}$, we denote by $\mathcal{K}_{r}^{T}(E, n)$ the set of functions $G:(0, T] \times \mathbf{R}^{d} \rightarrow \mathbf{D}^{n, \infty}(E)$ satisfying the following:

1. $G(t, \cdot)$ is $n$-times continuously differentiable and $\left[\partial^{\alpha} G / \partial x^{\alpha}\right]$ is continuous in $(t, x) \in(0, T] \times \mathbf{R}^{d}$ a.s. for any multi-index $\alpha$ of the elements of $\{1, \cdots, d\}$ with length $|\alpha| \leq n$.

2. For all $k \leq n-|\alpha|, p \in[1, \infty)$,

$$
\sup _{t \in(0, T], x \in \mathbf{R}^{d}} t^{-r / 2}\left\|\frac{\partial^{\alpha} G}{\partial x^{\alpha}}(t, x)\right\|_{\mathbf{D}^{k, p}}<\infty .
$$

We write $\mathcal{K}_{r}^{T}$ for $\mathcal{K}_{r}^{T}(\mathbf{R}, \infty)$ 
The properties of the Kusuoka-Stroock functions are the following. (See Lemma 5.1.2 of Nee (2010) or Lemma 75 of Crisan et al. (2013) for the proof.)

Lemma 4.1 [Properties of Kusuoka-Stroock functions]

1. The function $(t, x) \in(0, T] \times \mathbf{R}^{d} \mapsto X_{t}^{x}$ belongs to $\mathcal{K}_{0}^{T}$, for any $T>0$.

2. Suppose $G \in \mathcal{K}_{r}^{T}(n)$ where $r \geq 0$. Then, for $i=1, \cdots, d$,

$$
\text { (a) } \int_{0}^{\cdot} G(s, x) d W_{s}^{i} \in \mathcal{K}_{r+1}^{T}(n) \text {, and }(b) \int_{0}^{\cdot} G(s, x) d s \in \mathcal{K}_{r+2}^{T}(n)
$$

3. If $G_{i} \in \mathcal{K}_{r_{i}}^{T}\left(n_{i}\right), i=1, \cdots, N$, then

$$
\text { (a) } \prod_{i}^{N} G_{i} \in \mathcal{K}_{r_{1}+\cdots+r_{N}}^{T}\left(\min _{i} n_{i}\right) \text {, and }(b) \sum_{i=1}^{N} G_{i} \in \mathcal{K}_{\min _{i} r_{i}}^{T}\left(\min _{i} n_{i}\right) \text {. }
$$

Next, we summarize the Malliavin's integration by parts formula using Kusuoka-Stroock functions. For any multi-index $\alpha^{(k)}:=\left(\alpha_{1}, \cdots, \alpha_{k}\right) \in\{1, \cdots, d\}^{k}, k \geq 1$, we denote by $\partial_{\alpha(k)}$ the partial derivative $\frac{\partial^{k}}{\partial x_{\alpha_{1}} \cdots \partial x_{\alpha_{k}}}$.

Proposition 4.1 Let $G:(0, T] \times \mathbf{R}^{d} \rightarrow \mathbf{D}^{\infty}=\mathbf{D}^{\infty, \infty}(\mathbf{R})$ be an element of $\mathcal{K}_{r}^{T}$ and let $f$ be a function that belongs to the space $C_{b}^{\infty}\left(\mathbf{R}^{d}\right)$. Then for any multi-index $\alpha^{(k)} \in\{1, \cdots, d\}^{k}, k \geq 1$, there exists $H_{\alpha^{(k)}}\left(X_{t}^{x}, G(t, x)\right) \in \mathcal{K}_{r-\mid \alpha}^{T}(k) \mid$ such that

$$
E\left[\partial_{\alpha^{(k)}} f\left(X_{t}^{x}\right) G(t, x)\right]=E\left[f\left(X_{t}^{x}\right) H_{\alpha^{(k)}}\left(X_{t}^{x}, G(t, x)\right)\right]
$$

with

$$
\left\|H_{\alpha^{(k)}}\left(X_{t}^{x}, G(t, x)\right)\right\|_{L^{p}} \leq C(T, x) t^{\left(r-\left|\alpha^{(k)}\right|\right) / 2},
$$

where $H_{\alpha^{(k)}}\left(X_{t}^{x}, G(t, x)\right)$ is recursively given by

$$
\begin{aligned}
H_{(i)}\left(X_{t}^{x}, G(t, x)\right) & =\delta\left(\sum_{j=1}^{N} G \gamma_{i j}^{X_{t}^{x}} D X_{t}^{x, j}\right), \\
H_{\alpha(k)}\left(X_{t}^{x}, G(t, x)\right) & =H_{\left(\alpha_{k}\right)}\left(X_{t}^{x}, H_{\alpha(k-1)}\left(X_{t}^{x}, G(t, x)\right)\right),
\end{aligned}
$$

and a positive constant $C(T, x)$ is depending on $T$ and $x$. Here, $\left(\gamma_{i j}^{X_{t}^{x}}\right)_{1 \leq i, j \leq n}$ is the inverse matrix of the Malliavin covariance of $X_{t}^{x}$.

Proof. Apply Corollary 3.7 of Kusuoka and Stroock (1984) and Lemma 8-(3) of Kusuoka (2003) with Proposition 2.1.4 of Nualart (2006).

Remark 4.1 Kusuoka (2003) shows that Proposition 4.1 holds under the UFG condition. See p. 262 of Kusuoka (2003) for the definition of the UFG condition. We remark that if the coefficients of the forward SDE satisfy the uniform Hörmander condition, then they satisfy the UFG condition. We also remark if the coefficients of the forward SDE satisfy the uniform ellipticity condition, then they satisfy the UFG condition.

\subsection{Asymptotic Expansions for the Expectation of Functional of the Solution to FSDE}

This subsection derives the asymptotic expansions for the expectations of the composite functionals of smooth test functions $\varphi \in C_{b}^{\infty}$ and the solution to the forward SDE (1). Hereafter, let us denote $X_{i, T}^{t, x, \varepsilon}$ by $\frac{1}{i !} \frac{\partial^{i}}{\partial \varepsilon^{i}} X_{T}^{t, x, \varepsilon}, i \in \mathbf{N}$. In the first place, we characterize the expansion of the solution to the SDE (1) as a Kusuoka-Stroock finction.

Lemma 4.2 For $s \in(t, T]$,

$$
X_{i, s}^{t, x, \varepsilon} \in \mathcal{K}_{i}^{T}, i \in \mathbf{N} .
$$

Proof. We prove the assertion by induction. First,

$$
\begin{aligned}
\frac{\partial}{\partial \varepsilon} X_{s}^{t, x, \varepsilon}=\sum_{j=1}^{d} & {\left[\int_{t}^{s} \nabla X_{s}^{t, x, \varepsilon}\left(\nabla X_{u}^{t, x, \varepsilon}\right)^{-1} \sigma_{j}\left(u, X_{u}^{t, x, \varepsilon}\right) d W_{u}^{j}\right.} \\
& \left.-\varepsilon \int_{t}^{s} \nabla X_{s}^{t, x, \varepsilon}\left(\nabla X_{u}^{t, x, \varepsilon}\right)^{-1} \nabla_{x} \sigma_{j}\left(u, X_{u}^{t, x, \varepsilon}\right) \sigma_{j}\left(u, X_{u}^{t, x, \varepsilon}\right) d u\right] .
\end{aligned}
$$

Since $\nabla X_{s}^{t, x, \varepsilon},\left(\nabla X_{u}^{t, x, \varepsilon}\right)^{-1} \in \mathcal{K}_{0}^{T}$ and $\sigma_{j}, j=1, \cdots, d$ are bounded, we have $\frac{\partial}{\partial \varepsilon} X_{s}^{t, x, \varepsilon} \in \mathcal{K}_{1}^{T}$ by using the properties 2 and 3 in Lemma 4.1 . 
For $i \geq 2, \frac{1}{i !} \frac{\partial^{i}}{\partial \varepsilon^{i}} X_{s}^{t, x, \varepsilon}=\left(\frac{1}{i !} \frac{\partial^{i}}{\partial \varepsilon^{i}} X_{s}^{t, x, \varepsilon, 1}, \cdots, \frac{1}{i !} \frac{\partial^{i}}{\partial \varepsilon^{i}} X_{s}^{t, x, \varepsilon, d}\right)$ is recursively determined by the following:

$$
\begin{aligned}
\frac{1}{i !} \frac{\partial^{i}}{\partial \varepsilon^{i}} X_{s}^{t, x, \varepsilon, l}= & \sum_{\mathbf{i}_{\beta}, \mathbf{d}^{(\beta)}}^{(i)} \int_{t}^{s}\left(\prod_{k=1}^{\beta} \frac{1}{i_{k} !} \frac{\partial^{i_{k}}}{\partial \varepsilon^{i_{k}}} X_{u}^{t, x, \varepsilon, d_{k}}\right) \partial_{\mathbf{d}^{(\beta)}} b^{l}\left(u, X_{u}^{t, x, \varepsilon}\right) d u \\
& +\sum_{\mathbf{i}_{\beta}, \mathbf{d}(\beta)}^{(i-1)} \int_{t}^{s}\left(\prod_{k=1}^{\beta} \frac{1}{i_{k} !} \frac{\partial^{i_{k}}}{\partial \varepsilon^{i_{k}}} X_{u}^{t, x, \varepsilon, d_{k}}\right) \sum_{j=1}^{d} \partial_{\mathbf{d}^{(\beta)} \sigma_{j}^{l}\left(u, X_{u}^{t, x, \varepsilon}\right) d W_{u}^{j}} \\
& +\varepsilon \sum_{\mathbf{i}_{\beta}, \mathbf{d}(\beta)}^{(i)} \int_{t}^{s}\left(\prod_{k=1}^{\beta} \frac{1}{i_{k} !} \frac{\partial^{i_{k}}}{\partial \varepsilon^{i_{k}}} X_{u}^{t, x, \varepsilon, d_{k}}\right) \sum_{j=1}^{d} \partial_{\mathbf{d}^{(\beta)}} \sigma_{j}^{l}\left(u, X_{u}^{t, x, \varepsilon}\right) d W_{u}^{j},
\end{aligned}
$$

where

$$
\sum_{\mathbf{i}_{\beta}, \mathbf{d}^{(\beta)}}^{(i)}:=\sum_{\beta=1}^{i} \sum_{\mathbf{i}_{\beta} \in L_{i, \beta}} \sum_{\mathbf{d}^{(\beta)} \in\{1, \cdots, d\}^{\beta}} \frac{1}{\beta !},
$$

and

$$
L_{i, \beta}:=\left\{\mathbf{i}_{\beta}=\left(i_{1}, \cdots, i_{\beta}\right) ; \sum_{k=1}^{\beta} i_{k}=i ;\left(i, i_{k}, \beta \in \mathbf{N}\right)\right\}
$$

The above SDEs is linear and the order of the Kusuoka-Stroock function $\frac{1}{i !} \frac{\partial^{i}}{\partial \varepsilon^{i}} X_{s}^{t, x, \varepsilon}$ is determined inductively by the term

$$
\sum_{\mathbf{i}_{\beta}, \mathbf{d}^{(\beta)}}^{(i-1)} \int_{t}^{s} \nabla X_{s}^{t, x, \varepsilon}\left(\nabla X_{u}^{t, x, \varepsilon}\right)^{-1}\left(\prod_{k=1}^{\beta} \frac{1}{i_{k} !} \frac{\partial^{i_{k}}}{\partial \varepsilon^{i_{k}}} X_{u}^{t, x, \varepsilon, d_{k}}\right) \sum_{j=1}^{d} \partial_{\mathbf{d}^{(\beta)}} \sigma_{j}\left(u, X_{u}^{t, x, \varepsilon}\right) d W_{s}^{j} \in \mathcal{K}_{i}^{T},
$$

Since this term gives the minimum order in the terms that consist of (88). Then, $\frac{1}{i !} \frac{\partial^{i}}{\partial \varepsilon^{i}} X_{s}^{t, x, \varepsilon} \in \mathcal{K}_{i}^{T}$ by using the properties 2 and 3 in Lemma 4.1 .

The next proposition presents precise evaluation of the asymptotic expansions for the expectations of $E\left[\varphi\left(X_{T}^{t, x, \varepsilon}\right)\right]$ and $E\left[\varphi\left(X_{T}^{t, x, \varepsilon}\right) N_{T}^{t, x, \varepsilon}\right] \sigma(t, x)$ for a given smooth function $\varphi$.

\section{Proposition 4.2}

1. For $\varphi \in C_{b}^{\infty}\left(\mathbf{R}^{d}\right)$, there exists a constant $C(N, T, x)$ depending on $N, T$ and $x$ such that

$$
\left|E\left[\varphi\left(X_{T}^{t, x, \varepsilon}\right)\right]-\left\{E\left[\varphi\left(\bar{X}_{T}^{t, x, 0}\right)\right]+\sum_{i=1}^{N} \varepsilon^{i} E\left[\varphi\left(\bar{X}_{T}^{t, x, 0}\right) \pi_{i, T}^{t, x}\right]\right\}\right| \leq \varepsilon^{N+1} C(N, T, x)(T-t)^{(N+2) / 2},
$$

where $\bar{X}_{T}^{t, x, 0}=X_{T}^{t, x, 0}+\varepsilon X_{1, T}^{t, x, 0}$ and

$$
\pi_{i, T}^{t, x}=\sum_{l_{k}, \alpha^{(k)}}^{(i)} H_{\alpha^{(k)}}\left(X_{1, T}^{t, x, 0}, \prod_{j=1}^{k} X_{l_{j}+1, T}^{t, x, 0, \alpha_{j}}\right), i=1, \cdots, N
$$

Here, we use the following notations:

$$
\begin{aligned}
X_{1, T}^{t, x, 0} & :=\left.\frac{\partial}{\partial \varepsilon} X_{T}^{t, x, \varepsilon}\right|_{\varepsilon=0}=\sum_{j=1}^{d} \int_{t}^{s} \nabla X_{s}^{t, x, 0}\left(\nabla X_{u}^{t, x, 0}\right)^{-1} \sigma_{j}\left(u, X_{u}^{t, x, 0}\right) d W_{u}^{j}, \\
X_{i, T}^{t, x, 0, \alpha_{j}} & :=\left.\frac{1}{i !} \frac{\partial^{i}}{\partial \varepsilon^{i}} X_{T}^{t, x, \varepsilon, \alpha_{j}}\right|_{\varepsilon=0} .
\end{aligned}
$$

2. For $\varphi \in C_{b}^{\infty}\left(\mathbf{R}^{d}\right)$, there exists $C$ depending on $N$, $T$ and $x$ such that

$$
\begin{aligned}
& \left|E\left[\varphi\left(X_{T}^{t, x, \varepsilon}\right) N_{T}^{t, x, \varepsilon}\right] \sigma(t, x)-\left\{E\left[\varphi\left(\bar{X}_{T}^{t, x, 0}\right) N_{0, T}^{t, x}\right] \sigma(t, x)+\sum_{i=1}^{N} \varepsilon^{i} E\left[\varphi\left(\bar{X}_{T}^{t, x, 0}\right) N_{i, T}^{t, x}\right] \sigma(t, x)\right\}\right| \\
& \leq \varepsilon^{N+1} C(N, T, x)(T-t)^{(N+2) / 2},
\end{aligned}
$$


where $\bar{X}_{T}^{t, x, 0}=X_{T}^{t, x, 0}+\varepsilon X_{1, T}^{t, x, 0} ; N_{0, T}^{t, x}=\left(N_{0, T}^{t, x, 1}, \cdots, N_{0, T}^{t, x, d}\right)$ and $N_{i, T}^{t, x}=\left(N_{i, T}^{t, x, 1}, \cdots, N_{i, T}^{t, x, d}\right), i=1, \cdots, N$ are given respectively by

$$
N_{0, T}^{t, x, k}=\sum_{j=1}^{d} H_{(j)}\left(\bar{X}_{T}^{t, x, 0}, \partial_{k} \bar{X}_{T}^{t, x, 0, j}\right), \quad 1 \leq k \leq d
$$

and

$$
N_{i, T}^{t, x, k}=\sum_{j=1}^{d} H_{(j)}\left(\bar{X}_{T}^{t, x, 0}, \partial_{k} \bar{X}_{T}^{t, x, 0, j} \pi_{i, T}^{t, x}\right)+\partial_{k} \pi_{i, T}^{t, x}, \quad 1 \leq k \leq d .
$$

Remark 4.2 The result 1 is almost same error order as in Lipschitz case. That is, for a Lipschitz function $\varphi$ on $\mathbf{R}^{d}$, there exists a constant $C(N, T, x)$ depending on $N, T$ and $x$ such that

$$
\left|E\left[\varphi\left(X_{T}^{t, x, \varepsilon}\right)\right]-\left\{E\left[\varphi\left(\bar{X}_{T}^{t, x, 0}\right)\right]+\sum_{i=1}^{N} \varepsilon^{i} E\left[\varphi\left(\bar{X}_{T}^{t, x, 0}\right) \pi_{i, T}^{t, x}\right]\right\}\right| \leq \varepsilon^{N+1} C(N, T, x)(T-t)^{(N+2) / 2} .
$$

However, in Lipschitz case, the expansion error for $E\left[\varphi\left(X_{T}^{t, x, \varepsilon}\right) N_{T}^{t, x, \varepsilon}\right] \sigma(t, x)$ is given by

$$
\begin{aligned}
& \left|E\left[\varphi\left(X_{T}^{t, x, \varepsilon}\right) N_{T}^{t, x, \varepsilon}\right] \sigma(t, x)-\left\{E\left[\varphi\left(\bar{X}_{T}^{t, x, 0}\right) N_{0, T}^{t, x}\right] \sigma(t, x)+\sum_{i=1}^{N} \varepsilon^{i} E\left[\varphi\left(\bar{X}_{T}^{t, x, 0}\right) N_{i, T}^{t, x}\right] \sigma(t, x)\right\}\right| \\
& \leq \varepsilon^{N+1} C(N, T, x)(T-t)^{(N+1) / 2} .
\end{aligned}
$$

We also remark that when $\varphi$ is a bounded Borel function (even if it is non-smooth), we have

$$
\begin{aligned}
& \left|E\left[\varphi\left(X_{T}^{t, x, \varepsilon}\right)\right]-\left\{E\left[\varphi\left(\bar{X}_{T}^{t, x, 0}\right)\right]+\sum_{i=1}^{N} \varepsilon^{i} E\left[\varphi\left(\bar{X}_{T}^{t, x, 0}\right) \pi_{i, T}^{t, x}\right]\right\}\right| \leq \varepsilon^{N+1} C(N, T, x)(T-t)^{(N+1) / 2}, \\
& \left|E\left[\varphi\left(X_{T}^{t, x, \varepsilon}\right) N_{T}^{t, x, \varepsilon}\right] \sigma(t, x)-\left\{E\left[\varphi\left(\bar{X}_{T}^{t, x, 0}\right) N_{0, T}^{t, x}\right] \sigma(t, x)+\sum_{i=1}^{N} \varepsilon^{i} E\left[\varphi\left(\bar{X}_{T}^{t, x, 0}\right) N_{i, T}^{t, x}\right] \sigma(t, x)\right\}\right| \\
& \leq \varepsilon^{N+1} C(N, T, x)(T-t)^{N / 2} .
\end{aligned}
$$

Proof. The proof mainly relies on Proposition 4.1 and Lemma 4.2.

1. $X_{T}^{t, x, \varepsilon}$ degenerates when $\varepsilon \downarrow 0$. Then, we define $F_{T}^{t, x, \varepsilon}$ as follows:

$$
F_{T}^{t, x, \varepsilon}:=\frac{X_{T}^{t, x, \varepsilon}-X_{T}^{t, x, 0}}{\varepsilon} .
$$

$F_{T}^{t, x, \varepsilon} \in \mathbf{D}^{\infty}$ is a non-degenerate Wiener functional by Assumption 2.1. Let $\delta_{y}(\cdot)$ be the delta function. $\delta_{y}\left(F_{T}^{t, x, \varepsilon}\right) \in \tilde{\mathbf{D}}^{-\infty}=\cup_{k \geq 0} \cap_{q \geq 1} \mathbf{D}^{-k, q}$ is expanded as follows:

$$
\begin{aligned}
\delta_{y}\left(F_{T}^{t, x, \varepsilon}\right)= & \delta_{y}\left(F_{T}^{t, x, 0}\right)+\left.\sum_{i=1}^{N} \frac{\varepsilon^{i}}{i !} \frac{\partial^{i}}{\partial \varepsilon^{i}} \delta_{y}\left(F_{T}^{t, x, \varepsilon}\right)\right|_{\varepsilon=0} \\
& +\left.\varepsilon^{N+1} \int_{0}^{1} \frac{(1-u)^{N}}{N !} \frac{\partial^{N+1}}{\partial \nu^{N+1}} \delta_{y}\left(F_{T}^{t, x, \nu}\right)\right|_{\nu=\varepsilon u} d u .
\end{aligned}
$$

Therefore, the density of $F_{T}^{t, x, \varepsilon}$ is calculated by the integration by parts:

$$
\begin{aligned}
p^{F^{\varepsilon}}(t, T, 0, y)= & E\left[\delta_{y}\left(F_{T}^{t, x, \varepsilon}\right)\right] \\
= & E\left[\delta_{y}\left(F_{T}^{t, x, 0}\right)\right]+\sum_{i=1}^{N} \varepsilon^{i} E\left[\delta_{y}\left(F_{T}^{t, x, 0}\right) \pi_{i, T}^{t, x}\right] \\
& +\varepsilon^{N+1} \int_{0}^{1}(1-u)^{N} E\left[\delta_{y}\left(F_{T}^{, t, x, \varepsilon u}\right) \tilde{\pi}_{N+1, T}^{t, x, \varepsilon u}\right] d u,
\end{aligned}
$$

where

$$
\tilde{\pi}_{N+1, T}^{t, x, \varepsilon u}=(N+1) \sum_{l_{k}, \alpha^{(k)}}^{(N+1)} H_{\alpha(k)}\left(X_{1, T}^{t, x, \varepsilon u}, \prod_{j=1}^{k} X_{l_{j}+1, T}^{t, x, \varepsilon u, \alpha_{j}}\right) .
$$


Then, we have

$$
\begin{aligned}
\int_{\mathbf{R}^{d}} \varphi(y) p^{\varepsilon}(t, T, x, y) d y= & \int_{\mathbf{R}^{d}} \varphi(y) p^{0}(t, T, x, y) d y+\sum_{i=1}^{N} \varepsilon^{i} \int_{\mathbf{R}^{d}} \varphi(y) E\left[\pi_{i, T}^{t, x} \mid \bar{X}_{T}^{t, x, 0}=y\right] p^{0}(t, T, x, y) d y \\
& +\varepsilon^{N+1} \int_{0}^{1}(1-u)^{N} \int_{\mathbf{R}^{d}} \varphi(y) E\left[\tilde{\pi}_{N+1, T}^{t, x, \varepsilon u} \mid X_{T}^{t, x, \varepsilon u}=y\right] p^{\varepsilon u}(t, T, x, y) d y d u, \quad(107)
\end{aligned}
$$

where $p^{\varepsilon}(t, T, x, y)$ stands for the density function of $X_{T}^{t, x, \varepsilon}$ and $p^{0}(t, T, x, y)$ is given by

$$
p^{0}(t, T, x, y)=\frac{1}{\left(2 \pi \varepsilon^{2}\right)^{d / 2} \operatorname{det}(\Sigma(t, T))^{1 / 2}} e^{-\frac{\left(y-X_{T}^{t, x, 0}\right) \Sigma^{-1}(t, T)\left(y-X_{T}^{t, x, 0}\right)^{\top}}{2 \varepsilon^{2}}},
$$

with the covariance matrix $\Sigma(t, T)$ of $F_{T}^{t, x, 0}$. For $N \in \mathbf{N}, 1 \leq k \leq N+1$, there exists $\zeta_{N+1, k, T}^{t, x, \varepsilon} \in \mathcal{K}_{N+1+k}^{T}$ such that

$$
E\left[\varphi\left(X_{T}^{t, x, \varepsilon}\right) \tilde{\pi}_{N+1, T}^{t, x, \varepsilon}\right]=\sum_{k=1}^{N+1} \sum_{\alpha(k) \in\{1, \cdots, d\}^{k}} E\left[\partial_{\alpha^{(k)}} \varphi\left(X_{T}^{t, x, \varepsilon}\right) \zeta_{N+1, k, T}^{t, x, \varepsilon}\right]
$$

Therefore, for some positive constants $C_{k}, k=1, \cdots, N+1$ and $C(N, T, x)$,

$$
\begin{aligned}
\left|E\left[\varphi\left(X_{T}^{t, x, \varepsilon}\right) \tilde{\pi}_{N+1, T}^{t, x, \varepsilon}\right]\right| & \leq \sum_{k=1}^{N+1} C_{k}\left\|\zeta_{N+1, k, T}^{t, x, \varepsilon}\right\|_{L^{1}} \\
& \leq C(N, T, x)(T-t)^{(N+2) / 2} .
\end{aligned}
$$

2. Differentiating $E\left[\varphi\left(X_{T}^{t, x, \varepsilon}\right)\right]$ with respect to $x$, we have

$E\left[\varphi\left(X_{T}^{t, x, \varepsilon}\right) N_{T}^{t, x, \varepsilon}\right]=E\left[f\left(\bar{X}_{T}^{t, x, 0}\right) N_{0, T}^{t, x}\right]+\sum_{i=1}^{N} \varepsilon^{i} E\left[\varphi\left(\bar{X}_{T}^{t, x, 0}\right) N_{i, T}^{t, x}\right]+\varepsilon^{N+1} \int_{0}^{1}(1-u)^{N} E\left[\varphi\left(X_{T}^{t, x, u \varepsilon}\right) \tilde{N}_{N+1, T}^{t, x, u \varepsilon}\right] d u(111)$

where $\tilde{N}_{N+1, T}^{t, x, \varepsilon}:=\left(\tilde{N}_{N+1, T}^{t, x, \varepsilon, 1}, \cdots, \tilde{N}_{N+1, T}^{t, x, \varepsilon, d}\right)$ and

$$
\tilde{N}_{N+1, T}^{t, x, \varepsilon, k}=\sum_{j=1}^{d} H_{(j)}\left(\bar{X}_{T}^{t, x, \varepsilon}, \partial_{k} \bar{X}_{T}^{t, x, \varepsilon, j} \tilde{\pi}_{N+1, T}^{t, x, \varepsilon}\right)+\partial_{k} \tilde{\pi}_{N+1, T}^{t, x, \varepsilon}
$$

We remark that there exists $\xi_{N+1, k, T}^{t, x, \varepsilon} \in \mathcal{K}_{N+1+k}^{T}$ such that

$$
E\left[\varphi\left(X_{T}^{t, x, \varepsilon}\right) \tilde{N}_{N+1, T}^{t, x, \varepsilon}\right]=\sum_{k=2}^{N+2} \sum_{\alpha^{(k)} \in\{1, \cdots, d\}^{k}} E\left[\partial_{\alpha^{(k)}} \varphi\left(X_{T}^{t, x, \varepsilon}\right) \xi_{N+1, k, T}^{t, x, \varepsilon}\right] .
$$

Therefore, for some positive constants $C_{k}, k=1, \cdots, N+1$ and $C(N, T, x)$,

$$
\begin{aligned}
\left|E\left[\varphi\left(X_{T}^{t, x, \varepsilon}\right) \tilde{N}_{N+1, T}^{t, x, \varepsilon}\right]\right| & \leq \sum_{k=1}^{N+1} C_{k}\left\|\xi_{N+1, k, T}^{t, x, \varepsilon}\right\|_{L^{1}} \\
& \leq C(N, T, x)(T-t)^{(N+2) / 2} .
\end{aligned}
$$

\section{Main result: Asymptotic Expansion of FBSDE}

This section finally derives our main result which is asymptotic expansions of $u^{\alpha, \varepsilon}(t, x)$ in $(9)$ and $\partial_{x} u^{\alpha, \varepsilon}(t, x) \sigma(t, x)$ in (10).

First, applying the Malliavin weights $\pi_{i, s}^{t, x}$ and $N_{i, s}^{t, x}, s \in(t, T], 1 \leq i \leq N$ in Proposition 4.2 with $p^{0}(t, s, x, y)$, $s \in(t, T]$ in (108), we define an approximation sequence for $\left(u^{0, \varepsilon}, \partial_{x} u^{0, \varepsilon} \sigma\right)$. Let $\left(u^{0, \varepsilon, N}, \partial_{x} u^{0, \varepsilon, N} \sigma\right)$ be

$$
u^{0, \varepsilon, N}(t, x):=\int_{\mathbf{R}^{d}} g(y)\left\{1+\sum_{i=1}^{N} \varepsilon^{i} E\left[\pi_{i, T}^{t, x} \mid \bar{X}_{T}^{t, x, 0}=y\right]\right\} p^{0}(t, T, x, y) d y
$$




$$
\begin{aligned}
\left(\partial_{x} u^{0, \varepsilon, N} \sigma\right)(t, x):= & \left(\partial_{x} u^{0, \varepsilon, N}(t, x)\right) \sigma(t, x) \\
=\int_{\mathbf{R}^{d}} g(y) E & {\left[N_{0, T}^{t, x} \mid \bar{X}_{T}^{t, x, 0}=y\right] p^{0}(t, T, x, y) d y \sigma(t, x) } \\
& +\sum_{i=1}^{N} \varepsilon^{i} \int_{\mathbf{R}^{d}} g(y) E\left[N_{i, T}^{t, x} \mid \bar{X}_{T}^{t, x, 0}=y\right] p^{0}(t, T, x, y) d y \sigma(t, x) .
\end{aligned}
$$

Also, for $n \in \mathbf{N}$ we define $\left(u_{n}^{0, \varepsilon, N}, \partial_{x} u_{n}^{0, \varepsilon, N} \sigma\right)$ as

$$
\begin{aligned}
u_{n}^{0, \varepsilon, N}(t, x) & :=E\left[\int_{t}^{T} F^{n}\left(r, t, x, 0, X_{r}^{t, x, 0}\right) d r\right]+\sum_{i=1}^{N} \varepsilon^{i} E\left[\int_{t}^{T} F^{n}\left(r, t, x, 0, X_{r}^{t, x, 0}\right) \pi_{i, r}^{t, x} d r\right] \\
& =\int_{t}^{T} \int_{\mathbf{R}^{d}} F^{n}(r, t, x, 0, y)\left\{1+\sum_{i=1}^{N} \varepsilon^{i} E\left[\pi_{i, r}^{t, x} \mid \bar{X}_{r}^{t, x, 0}=y\right]\right\} p^{0}(t, r, x, y) d y d s,
\end{aligned}
$$

and

$$
\begin{aligned}
& \partial_{x} u_{n}^{0, \varepsilon, N} \sigma(t, x)=E\left[\int_{t}^{T}\left[F^{n}\left(r, t, x, 0, X_{r}^{t, x, 0}\right)\right] N_{0, r}^{t, x} d r\right] \sigma(t, x)+\sum_{i=1}^{N} \varepsilon^{i} E\left[\int_{t}^{T}\left[F^{n}\left(r, t, x, 0, X_{r}^{t, x, 0}\right)\right] N_{i, r}^{t, x} d r\right] \sigma(t, x) \\
= & \int_{t}^{T} \int_{\mathbf{R}^{d}} F^{n}(r, t, x, 0, y)\left\{E\left[N_{0, r}^{t, x} \mid \bar{X}_{r}^{t, x, 0}=y\right]+\sum_{i=1}^{N} \varepsilon^{i} E\left[N_{i, r}^{t, x} \mid \bar{X}_{r}^{t, x, 0}=y\right]\right\} p^{0}(t, r, x, y) d y d s \sigma(t, x),
\end{aligned}
$$

where $F^{n}$ is defined as (32) and (33) in Theorem 3.1.

Then, setting each $g$ and $F^{n}$ as $\varphi$ in Proposition 4.2 , we obtain the following result.

Corollary $\mathbf{5 . 1}$ It holds that:

$$
\begin{array}{r}
\left\|u^{0, \varepsilon}-u^{0, \varepsilon, N}\right\|_{H_{\beta, \mu, T}}^{2} \leq \varepsilon^{2(N+1)} C(N, T), \\
\left\|\partial_{x} u^{0, \varepsilon} \sigma-\partial_{x} u^{0, \varepsilon, N} \sigma\right\|_{H_{\beta, \mu, T}}^{2} \leq \varepsilon^{2(N+1)} C(N, T),
\end{array}
$$

and that for each $n, N \in \mathbf{N}$,

$$
\begin{array}{r}
\left\|u_{n}^{0, \varepsilon}-u_{n}^{0, \varepsilon, N}\right\|_{H_{\beta, \mu, T}}^{2} \leq \varepsilon^{2(N+1)} C(N, T), \\
\left\|\partial_{x} u_{n}^{0, \varepsilon} \sigma-\partial_{x} u_{n}^{0, \varepsilon, N} \sigma\right\|_{H_{\beta, \mu, T}}^{2} \leq \varepsilon^{2(N+1)} C(N, T),
\end{array}
$$

where $C(N, T)$ stands for a generic constant depending on $N, T$.

Finally, combining Theorem 3.1. and Corollary 5.1 above, we state our main theorem, which shows expansions of $u^{\alpha, \varepsilon}(t, x)$ and $\partial_{x} u^{\alpha, \varepsilon}(t, x) \sigma(t, x)$ in terms of the perturbation parameters of the driver $\alpha$ and the forward SDE $\varepsilon$.

Theorem 5.1 For any $M, N \in \mathbf{N}$, there exist generic constants $C(M, T)$ depending on $M, T$ and $C(M, N, T)$ depending on $M, N, T$ such that

$$
\begin{aligned}
& \left\|u^{\alpha, \varepsilon}-\left\{u^{0, \varepsilon, N}+\sum_{i=1}^{M} \alpha^{i} u_{i}^{0, \varepsilon, N}\right\}\right\|_{H_{\beta, \mu, T}}^{2}+\left\|\partial_{x} u^{\alpha, \varepsilon} \sigma-\left\{\partial_{x} u^{0, \varepsilon, N} \sigma+\sum_{i=1}^{M} \alpha^{i} \partial_{x} u_{i}^{0, \varepsilon, N} \sigma\right\}\right\|_{H_{\beta, \mu, T}}^{2} \\
\leq & \alpha^{2(M+1)} C(M, T)+\varepsilon^{2(N+1)} C(M, N, T) .
\end{aligned}
$$

Proof. We have the following inequality:

$$
\begin{aligned}
& \quad\left\|u^{\alpha, \varepsilon}-\left\{u^{0, \varepsilon, N}+\sum_{i=1}^{M} \alpha^{i} u_{i}^{0, \varepsilon, N}\right\}\right\|_{H_{\beta, \mu, T}}^{2}+\left\|\partial_{x} u^{\alpha, \varepsilon} \sigma-\left\{\partial_{x} u^{0, \varepsilon, N} \sigma+\sum_{i=1}^{M} \alpha^{i} \partial_{x} u_{i}^{0, \varepsilon, N} \sigma\right\}\right\|_{H_{\beta, \mu, T}}^{2} \\
& \leq\left\|u^{\alpha, \varepsilon}-\left\{u^{0, \varepsilon}+\sum_{i=1}^{M} \alpha^{i} u_{i}^{0, \varepsilon}\right\}\right\|_{H_{\beta, \mu, T}}^{2} \\
& +\left\|\left\{u^{0, \varepsilon}+\sum_{i=1}^{M} \alpha^{i} u_{i}^{0, \varepsilon}\right\}-\left\{u^{0, \varepsilon, N}+\sum_{i=1}^{M} \alpha^{i} u_{i}^{0, \varepsilon, N}\right\}\right\|_{H_{\beta, \mu, T}}^{2} \\
& +\left\|\partial_{x} u^{\alpha, \varepsilon} \sigma-\left\{\partial_{x} u^{0, \varepsilon} \sigma+\sum_{i=1}^{M} \alpha^{i} \partial_{x} u_{i}^{0, \varepsilon} \sigma\right\}\right\|_{H_{\beta, \mu}, T}^{2}
\end{aligned}
$$




$$
+\left\|\left\{\partial_{x} u^{0, \varepsilon} \sigma+\sum_{i=1}^{M} \alpha^{i} \partial_{x} u_{i}^{0, \varepsilon} \sigma\right\}-\left\{\partial_{x} u^{0, \varepsilon, N} \sigma+\sum_{i=1}^{M} \alpha^{i} \partial_{x} u_{i}^{0, \varepsilon, N} \sigma\right\}\right\|_{H_{\beta, \mu, T}}^{2} .
$$

By Theorem 3.1. and Corollary 5.1 we have the statement.

\section{References}

[1] D. Crisan and F. Delarue, Sharp Derivative Bounds for Solutions of Degenerate Semi-linear Partial Differential Equations, Journal of Functional Analysis, 263, 3024-3101, (2012).

[2] D. Crisan, K. Manolarakis and C. Nee, Cubature Methods and Applications, preprint (2013).

[3] N. El Karoui, S.G. Peng and M.C. Quenez, Backward stochastic differential equations in finance, Math. Finance, 7 1-71. (1997).

[4] M. Fujii and A. Takahashi, Analytical Approximation for Non-linear FBSDEs with Perturbation Scheme, International Journal of Theoretical and Applied Finance, Vol.15-5, (2012a).

[5] M. Fujii and A. Takahashi, Perturbative Expansion of FBSDE in an Incomplete Market with Stochastic Volatility, Quarterly Jornal of Finance, Vol.2, No.3 (2012b).

[6] M. Fujii and A. Takahashi, Perturbative Expansion Technique for Non-linear FBSDEs with Interacting Particle Method, CARF-F-278, April, (2012c).

[7] M. Fujii, S. Sato and A. Takahashi, An FBSDE Approach to American Option Pricing with an Interacting Particle Method, CARF-F-302, November, (2012).

[8] S. Kusuoka, Malliavin Calculus Revisited, J. Math. Sci. Univ. Tokyo, 261-277, (2003).

[9] S. Kusuoka and D. Stroock, Applications of the Malliavin Calculus Part I, Stochastic Analysis (Katata/Kyoto 1982) 271-306 (1984)

[10] J. Ma and J. Zhang, Representation Theorem of Backward Stochastic Differential Equations, Annals of Applied Probability, Vol.12, No.4, 1390-1318, (2002).

[11] D. Nualart, The Malliavin Calculus and Related Topics, Springer, (2006).

[12] C. Nee, Sharp Gradient Bounds for the Diffusion Semigroup, Ph.D thesis in Imperial College London, (2011).

[13] A. Takahashi. and T. Yamada, An Asymptotic Expansion with Push-Down of Malliavin Weights, SIAM Journal on Financial Mathematics, Volume 3, pp 95-136, (2012).

[14] A. Takahashi. and T. Yamada, An Asymptotic Expansion for Forward-Backward SDEs: A Malliavin Calculus Approach, CARF-F-296, Submitted, (2012).

[15] A. Takahashi. and T. Yamada, On Error Estimates for Asymptotic Expansions with Malliavin Weights Application to Stochastic Volatility Model-, Preprint, CARF-F-324 (2013)

[16] J. Zhang, Some Fine Properties of Backward Stochastic Differential Equations, Ph.D thesis in Purdue University (1999). 